[SUNY BING 7/1/03 v4]

hep-ph/0308089

\title{
COHERENT PRODUCTION OF PAIRS OF PARABOSONS OF ORDER 2
}

\author{
Nicholas Frascino and Charles A. Nelson ${ }^{1}$ \\ Department of Physics, State University of New York at Binghamton \\ Binghamton, N.Y. 13902
}

\begin{abstract}
A parameter-free statistical model is used to study multiplicity signatures for coherent production of charged-pairs of parabosons of order $p=2$ in comparison with those arising in the case of ordinary bosons, $p=1$. Two non-negative real parameters arise because " $a b$ " and " $b a$ " are fundamentally distinct pair operators of charge ' +1 ', A-quanta and charge ' -1 ', B-quanta parabosons. In 3D plots of $P_{m}(q) \equiv$ " the probability of $m$ paraboson charged-pairs $+q$ positive parabosons" versus $\langle n\rangle$ and $\left\langle n^{2}\right\rangle$, the $p=1$ curve is found to lie on the relatively narrow $2 \mathrm{D} p=2$ surface.
\end{abstract}

\footnotetext{
${ }^{1}$ Electronic address: cnelson @ binghamton.edu
} 


\section{Introduction:}

This paper is a theoretical study of multiplicity signatures in coherent production of charged-pairs of parabosons [1-3] of order $p=2$ in comparison with those arising in the case of ordinary bosons, $p=1$. The investigation is partially motivated by phenomenological coherence analyses, circa 1970, of inelastic $\pi^{+} \pi^{-}$pair production from fixed targets with laboratory kinetic-energies up to $27 \mathrm{GeV}$ by C.P. Wang [4], and by Horn and Silver[5]. The present analysis is possible because the conserved-charge boson coherent states of $[5,6]$ are analogous to ones recently constructed for order $p=2$ parabosons [7]. One physical consequence of "order $p=2$ " is that two or less such bosons can occupy a totally anti-symmetric state. Appendix 1 contains a brief review of $p=2$ paraboson statistics.

Both the $p=2$ and $p=1$ models considered in this paper are notably "statistical" and crudely "unrealistic" in being free of kinematic and dynamical parameters associated with physically important production quantities such as the distributions of available energy/momentum, the kinematic size and other characteristics of the production region, resonances, masses and other conserved quantum numbers (e.g. isotopic spin in the $p=1$ case [8]), because only the $U(1)$ charge conservation constraint has been imposed. Consequently, unlike what some readers would expect the situation to be from consideration of other particle-production models, in the figures the relative sizes of different aspects of the peaks, and of other structures, of the surfaces/curves cannot be adjusted without additional assumptions and complications. So despite the authors efforts, an interested viewer must sometimes force oneself to "look" in examination of some aspects

of the figures. In the $p=1$ case, more realistic models/arguments were made [8] to investigate such issues as independent-emission and coherence in $\pi^{+} \pi^{-}$pair production but these analyses 
are by now, of course, extremely primitive versus the current very sophisticated computational treatments $[9,10]$ of multi-particle production in high-energy collisions. For instance, even in electron-positron collisions a wide variety of coherence phenomena such as stage-one and stage-two spin-correlations in fermion-antifermion pair production, Bose-Einstein interference effects, and color-reconnection effects are used in various computational frameworks to describe and investigate particle production at LEP. Despite the lack of inclusion of other physics that would be required in a realistic application, we think that the focus in this paper on a comparison of these parameter-free statistical models is interesting and instructive with respect to $p=2$ versus $p=1$ parastatistics, and to the associated coherences involved in the production of conserved-charge paraboson pairs.

From the then available data, in [4] Wang reported a regularity when, for various laboratory kinetic energies of the incoming primary particle, the relative frequency of events $P_{m}(q)$ was plotted against the mean multiplicty $<n>$ for charged $\pi^{+} \pi^{-}$pairs for $p p, \pi^{ \pm} p, p n, \pi^{ \pm} n$, and $n n$ collisions. The data set was a compilation of about 50 inelastic production experiments with incoming primary kinetic energies extending from below production threshold to $27 \mathrm{GeV}$. Wang tried to fit the regularity with two Poisson-type distributions. In [5], Horn and Silver constructed instead a simple parameter-free statistical model and found that, ignoring fluctuations in the data due to the specific structure of the various channels, the model did agree $[5,7]$ with the universal trends of the experimental regularity reported by Wang. One construction of the Horn-Silver model is/was from the $p=1$ conserved-charge coherent states. Versus the inelastic reactions' multiplicity data, in [5] Horn and Silver argued for a statistical treatment of the gross features because (i) momentum conservation should be a weak constraint since the emitted pions occupy a small part of the available phase space, (ii) total isospin conservation on the distribution of charged 
pions should also be weak since neutral pions are summed over, and so (iii) charge conservation remains as the important constraint. They derived the $P_{m}(q)$ distribution discussed below. Their $P_{m}(0)$ distribution, i.e. the percentage of events with "m" $\pi^{+} \pi^{-}$pairs and no extra $\pi^{ \pm}$'s , had been considered earlier by Kastrup [11].

At the present time, the only way known to construct the analogous model in the $p=2$ case is to use conserved-charge coherent states. An alternative formulation with respect to either the statistical aspects or the coherence aspects is not currently available. So we now briefly digress to discuss what are these conserved-charge coherent states and then compare them with their $p=1$ counterpart:

A charged-paraboson pair in order $p=2$ consists of one A quanta of charge ' +1 ' and one B quanta of charge '-1'. The Hermitian charge operator is defined by

$$
Q=N_{a}-N_{b}
$$

where $N_{a, b}$ are the parabose number operators, see Appendix 1. Although $Q$ does not commute with $a$ or $b$, and although the paraboson pair operators $a b \neq b a$, since

$$
[Q, a b]=0, \quad[Q, b a]=0, \quad[a b, b a]=0
$$

the $p=2$ coherent state can be defined as simultaneous eigenstate of $Q, a b$, and $b a$ :

$$
Q\left|q, z, z^{\prime}>=q\right| q, z, z^{\prime}>, \quad a b\left|q, z, z^{\prime}>=z\right| q, z, z^{\prime}>, \quad b a\left|q, z, z^{\prime}>=z^{\prime}\right| q, z, z^{\prime}>
$$

Note that unlike in the $p=1$ case (see below), here two complex numbers $z$ and $z^{\prime}$ arise because $a b$ and $b a$ are fundamentally distinct operators. Consequently, in the following multiplicity considerations, two non-negative parameters occur which are the moduli of these two complex numbers, 
$u \equiv|z|$ and $v \equiv\left|z^{\prime}\right|$. The explicit expressions for $\mid q, z, z^{\prime}>$ which involve the modified Bessel functions $I_{\left[\frac{q}{2}\right]}(u)$ and $I_{\left[\frac{q+1}{2}\right]}(v)$ are given in [7] for $q \geq 0$ and $q<0$; note $\left[\frac{q}{2}\right]=$ "integer part of $\frac{q}{2}$ ". For $q \geq 0$, the $M$ th-moment of the mean multiplicity for a $p=2$ charged-paraboson pair is $<n^{M}>\equiv<q, z, z^{\prime}\left|\left(N_{b}\right)^{M}\right| q, z, z^{\prime}>$. For $q$ fixed, the percentage of events with $m$ such pairs, $P_{m}(q)$, is the square of the moduli of the expansion coefficients of $\mid q, z, z^{\prime}>$ in terms of the two-mode parabose number Fock states ( see [7] ). The explicit multiplicity formulas corresponding to the figures shown in this paper can be found below in Section 2.

The simpler $p=1$ conserved-charge coherent state $\mid q, \xi>$ satisfies $Q|q, \xi>=q| q, \xi>$ and $a b|q, \xi>=\xi| q, \xi>$. Since $a b=b a$ for $p=1, \mid q, \xi>$ involves a single complex number $\xi$, and so the Horn-Silver multiplicity analysis involved a single, non-negative real parameter $x \equiv|\xi|$.

Because the $p=2$ construction involves two non-negative real parameters $u$ and $v$, we consider a 3D plot of $P_{m}(q)=Z(u, v)$ versus the quarter plane defined by $<n>\equiv<n_{b}>=X(u, v)$ and $<n^{2}>\equiv<\left(n_{b}\right)^{2}>=Y(u, v)$. This is the obvious generalization of Wang's plot and it is the focus of Section 2 and its figures. Section 3 contains some additional multiplicity formulas which are similar in structure for $p=1,2$. Section 4 contains a brief discussion of some of what has/hasn't been learned from this analysis of coherent production of charged-pairs of parabosons of order $p=2$. Appendix 2 and its figures are focused on how different shaped $\{u, v\}$ charts, or coordinate patches, appear when mapped onto the two-dimensional surface.

\section{$2 \quad P_{m}(q)$ versus $<n>,<n^{2}>$ :}

The analysis in this section is straight-forward because there are simple explicit mathematical formulas involving modified Bessel functions and because "Mathematica" [12] notebooks are con- 
venient for displaying the parametric 1-parameter $p=1$ "curve" and 2-parameter $p=2$ "varyingwidth, folded ribbon." We find that a concise "curve/ribbon" terminology is useful and appropriate for describing, for example, Figs. 1a,b, which are for $m=2$, and $q=1$. On a 2D side of the 3D display, the $p=2$ ribbon consequently appears projected as a relatively narrow, two-dimensional region which will be denoted as a "band"; see Fig. 1b which shows the front XZ side of Fig. 1a. In spite of their being from the more complex $u \leftrightarrow v$ asymmetric case, these two lead figures have been chosen in order to ab initio discuss some details and caveats.

If the reader initially finds Figs. 1a,b and their description too complex, the reader might go directly to Figs. 2 and 3a,b which are for the simpler $u \leftrightarrow v$ symmetric case.

In Figs. 1a,b, and in the remaining figures in this paper, the solid line is the $p=1$ curve. In these two figures, this $p=1$ curve is also the $\{0, v\}$ line on the $p=2$ ribbon. Near the peak, the "fold" is the bottom edge of the ribbon. As shown, the $p=2$ ribbon consists of open-circles for the non-folded $u \geq v$ region, and of solid-circles for the folded $u \leq v$ region. The upper edge of the ribbon is the $\{u, 0\}$ set of points. Slightly to the right of the peak, one can see from the solid-circles that each line of dots traveling leftward down the page, bends under (or "over", whichever as the viewer prefers) the fold to reach the $\{0, v\}$ line on the ribbon.

For $q$ odd, we assume it is a "complete fold". The words "complete fold" mean that one part of the $\{u, v\}$ ribbon lies exactly on top of another part, and so there is no difference in going "under" or "over" such a fold.

Due to the very limited analytic results, mental/visual care is generally advisable: (i) Only for the cases of $q=0$ and for $q=1$ is it proven that the $p=1$ curve always lies on the $p=2$ two dimensional surface. In these two cases the proof appears un-instructive for then the parametric- 
solution $u=0, v=2 x$ ( and for $q=0$ also $u=2 x, v=0$ ) avoids confronting different ratios of modified Bessel functions. For an arbitrary $q$ value, from the asymptotic limits for the modified Bessel functions, it follows that as $x \rightarrow 0$, the curve and as $u, v \rightarrow 0$, the ribbon must begin at the same point: the origin for $m \neq 0$ and $P_{0}(q)=1$ for $m=0$. Similarly, for large parameters for $\operatorname{arbitrary} q$, the curve and ribbon will also be at the same $3 \mathrm{D}$ point with $P_{m}(q) \rightarrow 0$, if $x=\frac{1}{2}(u+v)$. (ii) Only for $q$ even is it known that there is a complete fold of the ribbon because then $P_{m}(q)$, $<n>$, and $\left\langle n^{2}>\right.$ are each $u \leftrightarrow v$ symmetric. For $q$ even, the fold is the line $u=v$, c.f. the simpler Figs. 2 and 3a,b.

The "line of dots traveling leftward down the page" discussed above for the peak region in Figs. 1a,b are a set of $\{u, v\}$ values from a unit-negative-slope diagonal in the $\{u, v\}$ domain, see last figure of this paper and its discussion Fig. 8a,b.

Second, the reader should be aware that the "dots" displayed to show the two-dimensional surface correspond to specific $\{u, v\}$ parameter values and that the associated $u_{1}, u_{2}, \ldots, v_{1}, v_{2}, \ldots$ values are equally spaced, c.f. Fig. 5a below where some $\{u, v\}$ coordinate values are shown. In some figures, $u_{2}-u_{1}$ is not equal to $v_{2}-v_{1}$. We find that a careful usage of a few un-connected "dots" does not mislead in displaying these two-dimensional surfaces. The "dots" should not be confused with a random generation of data points, such as in a scatter plot. Note that the $u$ and $v$ parameters do not map into an orthogonal coordinate chart on the ribbon. In general, at each point on the ribbon the curvature is non-zero.

Third, in consideration of Figs. 1a,b, as well as the other figures in this paper, it should be noted that, even near the peak, the width of the ribbon is narrow versus, for instance, the halfwidth of the $p=1$ peak. This fact, besides the folding and the role of the $u$ and $v$ parameters, 
would be an important issue in attempting empirically to distinguish coherent production pairs of $p=2$ parabosons versus those of ordinary $p=1$ bosons. On the other hand, the ribbon width is indeed non-zero and to the eye there is generally a systematic above, or below, displacement of the visual center of the $p=2$ "band" from the $p=1$ curve as in Figs. 1a,b.

For the $m=2$, and $q=1$ case shown in Figs. 1a,b the formulas for the $p=1$ curve are

$$
\begin{array}{r}
<n>^{(1)}=\frac{x I_{2}(2 x)}{I_{1}(2 x)}, \quad<n^{2}>^{(1)}=<n>^{(1)}+\frac{x^{2} I_{3}(2 x)}{I_{1}(2 x)} \\
P_{2}^{(1)}(1)=\frac{x^{5}}{12 I_{1}(2 x)}
\end{array}
$$

and for the $p=2$ ribbon are

$$
\begin{array}{r}
<n>^{(2)}=\frac{1}{2}\left(\frac{u I_{1}(u)}{I_{0}(u)}+\frac{v I_{2}(v)}{I_{1}(v)}\right) \\
<n^{2}>^{(2)}=<n>^{(2)}+\frac{1}{4}\left(\frac{u^{2} I_{2}(u)}{I_{0}(u)}+\frac{2 u v I_{1}(u) I_{2}(v)}{I_{0}(u) I_{1}(v)}+\frac{v^{2} I_{3}(v)}{I_{1}(v)}\right) \\
P_{2}^{(2)}(1)=\frac{3 u^{4} v+6 u^{2} v^{3}+v^{5}}{384 I_{0}(u) I_{1}(v)}
\end{array}
$$

As in (4-8), a superscript "(1) or (2)", for $p=1$ or $p=2$, can be respectively written on $\langle n>$, $<n^{2}>$, and $P_{m}(q)$ when needed to avoid confusion. Usually one knows the $p$ order from the working context, and so these superscripts can often be suppressed.

Before discussing other specific $m$ and $q$ cases, we consider the parametric formulas for an arbitrary curve and ribbon.

\section{$2.1 \quad p=1$ curve:}

The $p=1$ curve $(X(x), Y(x), Z(x))$ is parametrized by the real non-negative parameter $x$. For $q$ $\geq 0$, in terms of modified Bessel functions, $I_{q}(2 x)$,

$$
X(x) \equiv<n>=\frac{x I_{q+1}(2 x)}{I_{q}(2 x)}
$$




$$
Y(x) \equiv<n^{2}>=<n>+\frac{x^{2} I_{q+2}(2 x)}{I_{q}(2 x)}
$$

Equivalently, $<n^{2}>=x^{2}\left(1-\frac{q I_{q+1}(2 x)}{x I_{q}(2 x)}\right)$ follows due to the recursion relation for $I_{q}$ 's. The probability for " $m$ boson pairs $+q$ positive bosons" is

$$
Z(x)=P_{m}(q)=\frac{x^{2 m+q}}{I_{q}(2 x) m !(m+q) !}
$$

For example, in the analysis of "ideal" data for the production of purely multi-pion final states, $P_{m}(q)$ would be the probability for the production of " $m+q$ " $\pi^{+}$'s and " $q$ " $\pi^{-}$'s. For $p=1$, it is instructive to consider the statistically-fundamental

$$
P_{m}(q)=\left(N_{q}\right)^{2} \frac{x^{2 m}}{m !(m+q) !}
$$

with an $m$-independent normalization constant $\left(N_{q}\right)^{-2}=x^{-q} I_{q}(2 x)$ because in this way one sees that it is via normalization that the modified Bessel function appears in development of the simple idea of charge-conservation-constrained Poisson distributions for independent $\pi^{+}$and $\pi^{-}$ production $[5,8]$.

For multi-pion final states Wang [1], and later Horn and Silver [2] in consideration of (12) as a model, plotted $P_{m}(q)$ versus $<n>$ for small values of $m$ and $q$. This is the $X Z$ plane, or "front" projection of the 3D figures considered in this paper.

\section{$2.2 p=2$ surface:}

The $p=2$ two-dimensional surface $(X(u, v), Y(u, v), Z(u, v))$ is parametrized by the two real nonnegative parameters $u$ and $v$. As for the $p=1$ curve discussed above, only the $Z$-coordinate is $m$ dependent. 
( $q$ Even): The case of $q$ even and $q \geq 0$ is symmetric in $u \leftrightarrow v$ with $Z(u, v)=Z(v, u)$, see below, and the $u \leftrightarrow v$ symmetric

$$
\begin{aligned}
& X(u, v) \equiv<n>^{(2)}=\frac{1}{2}\left(\frac{u I_{\frac{q+2}{2}}(u)}{I_{\frac{q}{2}}(u)}+\frac{v I_{\frac{q+2}{2}}(v)}{I_{\frac{q}{2}}(v)}\right) \\
& Y(u, v) \equiv<n^{2}>^{(2)}=<n>^{(2)}+\frac{1}{4}\left(\frac{u^{2} I_{\frac{q+4}{2}}(u)}{I_{\frac{q}{2}}(u)}+2 u v \frac{I_{\frac{q+2}{2}}(u)}{I_{\frac{q}{2}}(u)} \frac{I_{\frac{q+2}{2}}(v)}{I_{\frac{q}{2}}(v)}+\frac{v^{2} I_{\frac{q+4}{2}}(v)}{I_{\frac{q}{2}}(v)}\right)
\end{aligned}
$$

( $q$ Odd): The case of $q$ odd and $q \geq 0$ is asymmetric in $u \leftrightarrow v$ with $Z(u, v) \neq Z(v, u)$, and the $u \leftrightarrow v$ asymmetric

$$
\begin{aligned}
& X(u, v) \equiv<n>^{(2)}=\frac{1}{2}\left(\frac{u I_{\frac{q+1}{2}}(u)}{I_{\frac{q-1}{2}}(u)}+\frac{v I_{\frac{q+3}{2}}(v)}{I_{\frac{q+1}{2}}(v)}\right) \\
& Y(u, v) \equiv<n^{2}>^{(2)}=<n>^{(2)}+\frac{1}{4}\left(\frac{u^{2} I_{\frac{q+3}{2}}(u)}{I_{\frac{q-1}{2}}(u)}+2 u v \frac{I_{\frac{q+1}{2}}(u)}{I_{\frac{q-1}{2}}(u)} \frac{I_{\frac{q+3}{2}}(v)}{I_{\frac{q+1}{2}}(v)}+\frac{v^{2} I_{\frac{q+5}{2}}(v)}{I_{\frac{q+1}{2}}(v)}\right)
\end{aligned}
$$

For $q \geq 0$, the probability for " $m$ paraboson pairs $+q$ positive parabosons" is

$$
\begin{aligned}
& Z(u, v) \equiv P_{m}^{(2)}(q)=\left(N_{q}^{(2)}\right)^{2} \sum_{i=1}^{m+1} \tilde{P}_{q, m ; i} \\
& \left(N_{q}^{(2)}\right)^{-2}=\left(\frac{u}{2}\right)^{-\left[\frac{q}{2}\right]} I_{\left[\frac{q}{2}\right]}(u)\left(\frac{v}{2}\right)^{-\left[\frac{q+1}{2}\right]} I_{\left[\frac{q+1}{2}\right]}(v)
\end{aligned}
$$

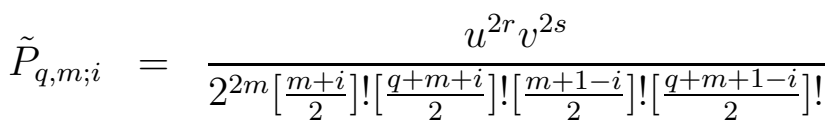

where in $\tilde{P}_{q, m ; i}$

$$
\begin{aligned}
r & \equiv\left[\frac{m-(-)^{q+m+i} i}{2}+\frac{1-(-)^{q}}{4}\right] \\
s & \equiv\left[\frac{m+(-)^{q+m+i} i}{2}+\frac{1+(-)^{q}}{4}\right]
\end{aligned}
$$

In paraboson statistics, it is sometimes convenient to use $[x]=$ "integer part of $x$ ", and $[x]$ ! = $[x][x-1] \cdots 1,[0] !=1$. Note that this $[x]$ symbol occurs in $(18-21)$. The use of the symbol $[x]$ enables a compactification of several analytic expressions and its use is convenient in computational notebooks. However, it is sometimes instructive, as in (13-16), to write out the various cases 
by letting $q=2 Q$, or $2 Q+1 ; Q=0,1, \cdots ;$ or similarly for $m$ or $i$. Throughout this paper, the appearance of "square braces" always denotes such an integer truncation. Note that the summation over "i" in (17) is also due to the fact that $a b \neq b a[7]$. The compact expressions are

$$
\begin{aligned}
& <n>^{(2)}=\frac{1}{2}\left(\frac{u I_{\left[\frac{q+2}{2}\right]}(u)}{I_{\left[\frac{q}{2}\right]}(u)}+\frac{v I_{\left[\frac{q+3}{2}\right]}(v)}{I_{\left[\frac{q+1}{2}\right]}(v)}\right) \\
& <n^{2}>^{(2)}=<n>^{(2)}+\frac{1}{4}\left(\frac{u^{2} I_{\left[\frac{q+4}{2}\right]}(u)}{I_{\left[\frac{g}{2}\right]}(u)}+2 u v \frac{I_{\left[\frac{q+2}{2}\right]}(u)}{I_{\left[\frac{q}{2}\right]}(u)} \frac{I_{\left[\frac{q+3}{2}\right]}(v)}{I_{\left[\frac{q+1}{2}\right]}(v)}+\frac{v^{2} I_{\left[\frac{q+5}{2}\right]}(v)}{I_{\left[\frac{q+1}{2}\right]}(v)}\right)
\end{aligned}
$$

Since from (17) the $p=2$ case non-trivially involves two parameters, $u$ and $v$, the figures in this paper are 3D ones with $P_{m}^{(p)}(q) \equiv Z^{(p)}$ plotted versus the two-dimensional $\langle n\rangle^{(p)} \equiv X^{(p)}$ and $<n^{2}>^{(p)} \equiv Y^{(p)}$ plane. Only the first octant is used.

\subsection{Other $m$ and $q$ cases:}

For the case $m=0, q=0$ the parametric expressions are very simple:

$$
\begin{gathered}
<n>^{(1)}=\frac{x I_{1}(2 x)}{I_{0}(2 x)}, \quad<n^{2}>^{(1)}=<n>^{(1)}+\frac{x^{2} I_{2}(2 x)}{I_{0}(2 x)} \\
P_{0}^{(1)}(0)=\frac{1}{I_{0}(2 x)} \\
<n>^{(2)}=\frac{1}{2}\left(\frac{u I_{1}(u)}{I_{0}(u)}+\frac{v I_{1}(v)}{I_{0}(v)}\right) \\
<n^{2}>^{(2)}=<n>^{(2)}+\frac{1}{4}\left(\frac{u^{2} I_{2}(u)}{I_{0}(u)}+\frac{2 u v I_{1}(u) I_{1}(v)}{I_{0}(u) I_{0}(v)}+\frac{v^{2} I_{2}(v)}{I_{0}(v)}\right) \\
P_{0}^{(2)}(0)=\frac{1}{I_{0}(u) I_{0}(v)}
\end{gathered}
$$

This is the simplest $u \leftrightarrow v$ symmetric case and the "right" $Y Z$-side is shown in Fig. 2. As previously mentioned, in the symmetric case, the fold is along $u=v$ which is shown by the dashed line in Fig. 2. The two edges of the original ribbon, $\{0, v\}$ and $\{u, 0\}$ are respectively mapped by 
$v=2 x, u=2 x$, into the $p=1$ curve. Only open-circle points from the $u \geq v$ region are shown. All $m=0$ curves/ribbons drop down from $P_{o}(q)=1$.

Figs. 3a,b, respectively show the 3D plot and the right $Y Z$-side for another symmetric case $m=1, q=0$. Note that, unlike in the preceding Fig. 2, in this case the $p=1$ curve now lies on the top of the ribbon until there is an almost $180^{\circ}$ twist, after the peak where the $p=1$ curve is on bottom. In Fig. $3 \mathrm{~b}$ the $Y Z$-side quite clearly shows this twist. The parametric expressions are respectively $P_{1}^{(1)}(0)=\frac{x^{2}}{I_{0}(2 x)}, P_{1}^{(2)}(0)=\frac{u^{2}+v^{2}}{4 I_{0}(u) I_{0}(v)}$.

In appendix 2 , there is a discussion of the $\{u, v\}$ coordinate charts associated with the asymmetric case $m=1, q=1$ shown in Figs. 4a - 4d. The parametric equations are respectively $P_{1}^{(1)}(1)=\frac{x^{3}}{2 I_{1}(2 x)}, P_{1}^{(2)}(1)=\frac{u^{2} v+v^{3}}{8 I_{0}(u) I_{1}(v)}$. [ The omitted figures for the asymmetric case $m=0, q=1$ similarly show the fold occurring in the $u \leq v$ region. ] Fig. 4a is the 3D display and Fig. 4b is the projection onto the front $X Z$ plane. Fig. $4 \mathrm{c}$ is from a different 3D viewpoint. It provides a view back into the origin, with a $Y X$ floor below and a $Y X$ ceiling above, in a "left-to-right, front axes labeling". This figure shows how nearly vertical the ribbon is. Lastly, Fig. 4d shows a close-up of the $m=1, q=1$ peak. This figure illustrates the fold. In it the upper solid line is the $p=1$ curve and the lower solid line is the $u=v$ curve which separates the open-circles $(u \geq v)$ from the solid-circles $(u \leq v)$.

The different shaped $\{u, v\}$ charts of Appendix 2 can be used in investigating the folding which occurs in a transverse crossing of the ribbon. Similarly, the lines $u=0$, also $v=0$ for $q$-odd, $u=v$, and for $q \geq 2$ also the independent $p=1$ curve, can be used. Both techniques are useful in studying what occurs in going out along the ribbon. In proceeding from the origin, or for $m=0$ from $P_{0}(q)=1$, there are twist(s) and a peak (absent for $m=0$ ). If as in Fig. 4c, the ribbon is 
viewed back in from the high $u$ and $v$ parametric coordinates, then in moving out from the origin but viewing back towards the origin, the almost $180^{\circ}$ twisting of the $u \geq v$ edge from the peak onward is counter-clockwise for $(m, q)=(1,0),(1,1),(2,1)$. For $(2,1),(2,2),(2,3)$ there is also a twist near the origin. For the peak in $(1,1)$, c.f. Fig. 4 a, the $Z$ coordinate values are: for the $v=0$ top edge, $Z(2.65,0)=0.475$; for the $p=1$ curve, $Z(0,3.25)=0.436$; for the $u=v$ curve $Z(1.85,1.85)=0.42$; and for the fold $Z(1.5,2.25)=0.41$.

\section{Additional Multiplicity Formulas:}

In the $p=2$ case, the $M$-th moment of the mean multiplicity

$$
<n_{b, a}^{M}>=\left(N_{q}\right)^{2}\left(\widehat{D_{b, a}}\right)^{M}\left(N_{q}\right)^{-2}
$$

where $\widehat{D_{b}}=\frac{1}{2}\left(u \frac{\partial}{\partial u}+v \frac{\partial}{\partial v}\right), \widehat{D_{a}}=\widehat{D_{b}}+q$. It follows that

$$
\begin{array}{r}
<n>=<n_{b}>=\left(N_{q}\right)^{2} S_{1}(u, v), \\
<n^{2}>=<n_{b}^{2}>=\left(N_{q}\right)^{2}\left(S_{2}+S_{1}\right), \\
<n_{b}^{3}>=\left(N_{q}\right)^{2}\left(S_{3}+3 S_{2}+S_{1}\right), \\
<n_{b}^{4}>=\left(N_{q}\right)^{2}\left(S_{4}+6 S_{3}+7 S_{2}+S_{1}\right), \\
<n_{b}^{5}>=\left(N_{q}\right)^{2}\left(S_{5}+10 S_{4}+25 S_{3}+15 S_{2}+S_{1}\right), \ldots
\end{array}
$$

where, with $\left(\begin{array}{c}M \\ l\end{array}\right)$ a binomial coefficient,

$$
\begin{aligned}
S_{M}(u, v) & =\bar{c} \sum_{l=0}^{M}\left(\begin{array}{c}
M \\
l
\end{array}\right)\left(\frac{u}{2}\right)^{M-l}\left(\frac{v}{2}\right)^{l} I_{\left[\frac{q+2(M-l)}{2}\right]}(u) I_{\left[\frac{q+1+2 l}{2}\right]}(v) \\
\bar{c} & =2^{\left[\frac{q}{2}\right]+\left[\frac{q+1}{2}\right]} u^{-\left[\frac{q}{2}\right]} v^{-\left[\frac{q+1}{2}\right]}
\end{aligned}
$$


The function $S_{M+1}(u, v)$ is generated by $\left(\widehat{D_{b}}-M\right) S_{M}(u, v)=S_{M+1}(u, v)$. The expression for $\left(N_{q}^{(2)}\right)^{2}$ is $(18)$ above.

Note that as in the $p=1$ case,

$$
<n_{a}^{M}>=\sum_{t=0}^{M}\left(\begin{array}{c}
M \\
t
\end{array}\right) q^{t}<n_{b}^{M-t}>
$$

When $q=0,<n_{a}^{M}>=<n_{b}^{M}>$, and for fixed $q,<n_{a}>=<n_{b}>+q$.

For $(q=-|q|)<0$, there are the following relations to the $q>0$ expressions:

$$
<n_{a}^{M}>_{-|q|}=<n_{b}^{M}>_{|q|}
$$

in (27-32) and exchange $u \leftrightarrow v$,

$$
<n_{b}^{M}>_{-|q|}=<n_{a}^{M}>_{|q|}
$$

in $(27,35)$ and exchange $u \leftrightarrow v$; and $P_{m}(|q|)=P_{m+|q|}(-|q|)$.

The analogous formulas for the $p=1$ case are

$$
<n_{b, a}^{M}>^{(1)}=\left(N_{q}^{(1)}\right)^{2}\left(\widehat{D_{b, a}^{(1)}}\right)^{M}\left(N_{q}^{(1)}\right)^{-2}
$$

where $\widehat{D_{b}^{(1)}}=\frac{1}{2}\left(x \frac{\partial}{\partial x}\right), \widehat{D_{a}^{(1)}}=\widehat{D_{b}^{(1)}}+q$. So $<n>^{(1)}=<n_{b}>^{(1)}=\left(N_{q}^{(1)}\right)^{2} s_{1}(x)$, $<n_{b}^{2}>^{(1)}=\left(N_{q}^{(1)}\right)^{2}\left(s_{2}+s_{1}\right), \ldots$ where

$$
s_{M}(x)=x^{M-q} I_{q+M}(x)
$$

The function $s_{M+1}(x)$ is generated by $\left(\widehat{D_{b}^{(1)}}-M\right) s_{M}(x)=s_{M+1}(x)$. The expression for $\left(N_{q}^{(1)}\right)^{2}$ is given after (12) above.

For $(q=-|q|)<0$ for $p=1,<n_{a}^{M}>_{-|q|}=<n_{b}^{M}>_{|q|},<n_{b}^{M}>_{-|q|}=<n_{a}^{M}>_{|q|}$, and $P_{m}(|q|)=P_{m+|q|}(-|q|)$. 


\section{Discussion:}

The $2 \mathrm{D}$ surface occurs in the $3 \mathrm{D}$ plots of the relative probabilities $P_{m}(q)$ versus $<n>,<n^{2}>$ as required by the coherences embodied in the equations in (3) for the $p=2$ conserved-charge coherent states. Unlike the analogous $p=1$ case, where such a model was motivated from a regularity in $\pi^{+} \pi^{-}$data, it is necessary in the case of the production of the charged-pairs of parabosons of order $p=2$ to assume an analogous coherent production mechanism and to assume a reasonable, but definite, treatment of the $a b$ and $b a$ operators. Neither of these two assumptions might be true if paraboson pairs are found to be produced in nature in high energy physics collisions, e.g. in central-diffactive-exchange experiments [14], or in some area of contemporary astrophysics/cosmology, e.g. in dark matter detectors [15] or by very high energy cosmic rays [16]. In the infra-red domains of QED and of QCD, coherent-state/degenerate-state coherence is a well-known phenomena. On the other hand in QCD, partonic jets dominate the very high energy hadronic production processes. In the case of the lower energy $\pi^{+} \pi^{-}$production, as was emphasized by Horn-Silver, and others, the situation is complex and there is much more physics and phenomenological structure than that incorporated in the reference $p=1$ model considered in this paper. In the approximation in which one neglects such additional physics, this $p=1$ model is parameter free because the $x$ parameter is effectively replaced by $<n>$, the mean number of final charged pairs. Similarly, the analogous $p=2$ model is parameter free for the $u$ and $v$ parameters are effectively replaced by $\left\langle n>\right.$ and $<n^{2}>$. At the present time, the existence and relative importance of other conserved quantum numbers, of resonances or other phenomenological interaction effects, etc. is completely unknown for a coherent production of pairs of parabosons of order 2. Nevertheless, with respect to experiments [14-16], one conclusion from this paper is 
that from a parameter-free statistical model, one would expect a signature of relatively narrow bands, due to projection of varying-width folded ribbons, will be present in 2D analysis of pair multiplicities from coherent production of parabosons of order 2.

In the case of $p=2$ parabosons, there will be a second kinematic variable [4] and multiplicity data will not scale in terms of a single-variable curve [11]. For instance, if the variable for the $p=1$ curve is $x \sim \frac{1}{2}(u+v)$, then for $p=2$ parabosons there will also be a dependence on $y \sim \frac{1}{2}(u-v)$. In Fig. 8a, with $x \sim \frac{1}{2}(u+v)$ fixed, the sensitivity to the second variable $y \sim \frac{1}{2}(u-v)$ is very striking and significantly greater than the naive width of the ribbon. In the region of the peak, Figs. $1 \mathrm{~b}$ and $4 \mathrm{~b}$ show that this signature is considerably enhanced when there is an extra positive paraboson, versus the case of only charged-paraboson-pairs, Fig. 3b. While an explicit dynamical production model is required to theoretically investigate the sensitivity with respect to specific kinematic variables, in independent-particle-emission models the total energy in the emitted particles is monotonically related to the intensity strength of the source. This suggests that two simple and useful kinematic variables are the sum, $E_{\text {total }}=E^{+}{ }_{\text {total }}+E^{-}$total, and difference $E^{+}{ }_{\text {total }}-E^{-}{ }_{\text {total }}$, of the total emitted $Q=1$ and $Q=-1$ energies.

The "identical particle" defining parabose tri-linear relations leave the two pair operators, $a b$ and $b a$, fundamentally distinct which (3) and present analysis maintain by the use of the two distinct $u$ and $v$ parameters. In the $p=1$ case, the $x^{2}=x_{+} x_{-}$parameter can be interpreted as a product of the intensity strengths of the $\pi^{+}$and $\pi^{-}$sources. In the $p=2$ case, $u$ and $v$ can be interpreted as the intensity strengths of the "ab" and " $b a$ " sources. Their difference is due to the two distinct orderings of the $a$ and $b$ operators. The physical observables are not always $u \leftrightarrow$ $v$ symmetric. In particular, the presence of a $q$ odd total charge produces an asymmetry in the 
folding of the 2D surface; for instance, see Eqs. $(15,16)$ and $(8)$.

There is overall $A^{+} \leftrightarrow B^{-}$symmetry ( $U(1)$ charge symmetry ): The "Probability for $(m+|q|)$ $A^{+}$'s and $(m) B^{-}$'s " equals "Probability for $(m) A^{+}$'s and $(m+|q|) B^{-}$'s ", because $P_{m}(|q|)=$ $P_{m+|q|}(-|q|)$ and $\left\langle n_{b}^{M}>_{-|q|}=<n_{a}^{M}>_{|q|}\right.$ for $M=$ integer.

The $U(1)$ charge might be a "hidden conserved-charge" such as to yield pure pair production, i.e. only final state events with $q=0$ would occur such as in the production of strange particles via the strong interactions. In this case, or by a designed selection of only pair final state events, the $p=2$ versus $p=1$ results of this paper can still be used with $\sum_{m=0}^{\infty} P_{m}(0)=1$. This is the simpler symmetric case and because of $q=0$ there would be more analytic control in such an analysis.

\section{Acknowledgments:}

One of us (CAN) thanks experimental and theoretical physicists for discussions. We thank Ted Brewster, Lorene Evans, Anthony Poole, and James L. Wolf for computer support. This work was partially supported by U.S. Dept. of Energy Contract No. DE-FG 02-86ER40291. 


\section{Appendix 1: Parabosons of order 2}

In local, relativistic quantum field theory, identical particles obey either (i) parabose and parafermi statistics [1-3] for which the number of particles in an antisymmetric or a symmetric state, respectively, cannot exceed a given integer $p$, or (ii) for two space dimensions, infinite statistics based on the braid group [13]. In parabose statistics, instead of bilinear, there are fundamental trilinear commutation relations:

$$
\begin{gathered}
{\left[a_{k},\left\{a_{l}^{\dagger}, a_{m}\right\}\right]=2 \delta_{k l} a_{m},\left[a_{k},\left\{a_{l}^{\dagger}, a_{m}^{\dagger}\right\}\right]=2 \delta_{k l} a_{m}^{\dagger}+2 \delta_{k m} a_{l}^{\dagger},} \\
{\left[a_{k},\left\{a_{l}, a_{m}\right\}\right]=0, \quad(k, l, m=1,2)}
\end{gathered}
$$

where $[C, D] \equiv C D-D C$, and $\{C, D\} \equiv C D+D C$. In the case of only two kinds of parabosons, there are some simple commutation relations between "A" paraboson operators and the "B" paraboson operators: letting $a \equiv a_{1}, b \equiv a_{2}$,

$$
\left[a, b^{2}\right]=0,\left[b, a^{2}\right]=0,\left[a^{\dagger}, b^{2}\right]=0,\left[b^{\dagger}, a^{2}\right]=0
$$

plus the hermitian conjugate relations. Order $p=2$ is simpler because there is the "self-contained set" of 3 relations $[2]$

$$
\begin{aligned}
& a_{m} a_{l} a_{k}^{\dagger}-a_{k}^{\dagger} a_{l} a_{m}=2 \delta_{k l} a_{m} \\
& a_{k} a_{l}^{\dagger} a_{m}-a_{m} a_{l}^{\dagger} a_{k}=2 \delta_{k l} a_{m}-2 \delta_{l m} a_{k} \\
& a_{k} a_{l} a_{m}-a_{m} a_{l} a_{k}=0
\end{aligned}
$$

The parabose number operators for $p=2$ order are defined by

$$
N_{a}=\frac{1}{2}\left\{a^{\dagger}, a\right\}-1, N_{b}=\frac{1}{2}\left\{b^{\dagger}, b\right\}-1
$$

In [7], the state-vector space for two-mode parabosons and the order $p=2$ conserved-charged parabose coherent states were constructed. 
Production and decay selection rules [2, 3] exclude known particles from obeying other [1] than the usual boson and fermion statistics, i.e. order $p=1$ parastatistics.

\section{Appendix 2: $\{u, v\}$ Charts on 2D surface}

In a determination of the statistical uncertainties for an application of the parametric formulas in Section 2 in comparison with actual experimental data, the structure(s) of the folded, partiallytwisted ribbons versus $p=1$ curves will enter and have to be correctly treated. For this reason, and for possible more abstract theoretical/mathematical use by other readers, the following details about how different shaped $\{u, v\}$ coordinate patches appear when mapped onto the 2D surface should be of interest. The lines $u=0$, also $v=0$ for $q$-odd, and $u=v$ provide useful standard references in examining what is taking place on each ribbon. In addition, for $q \geq 2$ the $p=1$ curve is a different reference since it no longer is the $u=0$ line.

The $m=1, q=1$ case which is $u \leftrightarrow v$ asymmetric is used in this appendix. This case is both generic and visually simple. Figs. 4a-4d were earlier displays of this case. The discussion begins with the non-folded $u \geq v$ region:

Figs. 5a,b show how the $u \geq v$ "strip" from $u=2$ to $u=3$ is mapped onto the peak region of the ribbon. In 3D Fig. 5a, the solid line that lies on the bottom of the "patch" on the ribbon is the $u=v$ set of points. The open-circle points on the top of the patch are the $v=0$ set of points. The points on the origin side in Fig. 5a are for the vertical $u=2$ line in Fig. 5b. The points on the patch farthest from the origin, which appear to have a nearly constant negative slope, are for the other vertical line in Fig. 5b, i.e. for $u=3$. Here there have been 5 sets of vertical points mapped from Fig. 5b onto the 5a patch on the ribbon.

Figs. 6a,b similarly show the differences when the $u \geq v$ points arise from three more widely 
separated constant- $u$ vertical lines with respectively $u \simeq 2,3,4$. To the left-side of the peak, $u \simeq 2$, the spacing between these constant- $u$-value points is rather small, whereas on the right of the peak, where $u \simeq 4$, the spacing between such points is significantly greater. The points are equally spaced in $v$ so there are more points taken at $u \simeq 4$ than at $u \simeq 2$.

Horizontal "strips" can similarly be used to study the folded $u \leq v$ region. However, a "ring" region or a "unit-negative-slope strip" region can be used which includes both $u \geq v$ and $u \leq v$ domains. Figs. 7a,b show what occurs when points in a "ring" of radii $r_{<}=3$ and $r_{>}=4$ are mapped onto the ribbon. In $7 \mathrm{a}$, the open-circle-dots are again from the simpler $u \geq v$ region, whereas the solid-dots are from the $u \leq v$ region where the fold occurs. Fig. 7c shows a close-up where the $u=0$ points ( $p=1$ curve) are on the upper solid curve; the $u=v$ points which divide the ring in half are on the lower solid curve. [ If instead, the points are from pie-slice-like sectors, the associated lines on the ribbon then extend indefinitely on out the ribbon. ]

Lastly, Fig. 8a,b show what happens when points are taken from a somewhat similar, but sometimes more useful, unit-negative-slope strip in the $u, v$ quadrant. In Fig. 8a the solid line is the $u=v$ curve. The "line of dots traveling leftward down the page" discussed in the text for the peak region in Figs. 1a,b are a set of $\{u, v\}$ values from such a unit-negative-slope diagonal. If instead, the points are taken from the orthogonal unit-positive-slope strips, the associated lines on the ribbon will start on a $\{0, v\}$ or $\{u, 0\}$ line, depending on which side of $u=v$ the strip is from, and then the line will extend indefinitely on out the ribbon. 


\section{References}

[1] H.S. Green, Phys.Rev. 90, 270 (1953); D.V. Volkov, Sov. Phys.-JETP, 11, 375 (1960); and c.f. W. Pauli, Handbuch der Physik Vol. 24, p. 113 of Sec. 14 (1934); p. 128-9 in A Scientific Biography of W. Pauli, C.P. Enz (2002)(Oxford U. Press).

[2] O.W. Greenberg and A.M.L. Messiah, Phys.Rev. 136, B248 (1964); ibid., 138, B1155 (1965).

[3] Y. Ohnuki and S. Kamefuchi, Quantum Field Theory and Parastatistics (1982) (Univ. Press of Tokyo).

[4] C.P. Wang, Phys.Rev. 180, 1463 (1969). Wang plotted $P_{m}(q)$ for $q=0$ versus the equivalent, mean number of charged prongs $<n_{s}>=2<n>+2=<n_{c}>$ (Horn-Silver). Figure 1a in Wang's paper shows the laboratory kinetic energies of the data points.

[5] D. Horn and R. Silver, Phys.Rev. D2, 2082 (1970).

[6] D. Bhaumik, K. Bhaumik, and B. Dutta-Roy, J. Phys. A: 9, 1507 (1976); J.R. Klauder and B.-S. Skagerstam, p. 43-48, Coherent States(1985) (World Sci. Pub. Co., Singapore); and see G.S. Agarwal, J. Opt. Soc. Am. B5, 1940 (1988).

[7] S. Jing and C.A. Nelson, J. Phys. A: 32, 4131 (1999).

[8] See, and references therein, D. Horn and R. Silver, Annals of Phys. (NY) 66, 509 (1971); D. Horn, Physics Rep. 4, 1 (1972); and D. Horn and F. Zachariasen, Hadron Physics at Very High Energies (1973) (W.A. Benjamin, Reading, MA).

[9] T. Sjostrand and M. Bengtssson, Comp. Phys. Comm. 43, 367 (1987); G. Marchesini and B.R. Webber, ibid. 67, 465 (1992); S. Jadach, Z. Was, R. Decker, and J.H. Kuhn, ibid. 76, 
361 (1993); S. Jadach, B.F.L. Ward, and Z. Was, ibid. 124, 233 (2000); B.F.L. Ward and S. Jadach, Acta Phys. Polon. B33, 1543 (2002); L. Lonnblad, Comp. Phys. Comm. 71, 15 (1992); and T. Sjostrand, P. Eden, C. Friberg, L. Lonnblad, G. Miu, S. Mrenna and E. Norrbin, ibid. 135, 238 (2001).

[10] See, and references therein, Perturbative Quantum Chromodynamics, ed. A.H. Mueller (1989) (World Sci. Pub. Co., Singapore); Yu L. Dokshitzer, V.A. Khoze, A.H. Mueller and S.I. Troyan, Basics of Perturbative QCD, ed. J. Tran Thanh Van (1991) (Gif-sur-Yvette, Editions Frontieres); J.C. Collins and D.E. Soper, Ann. Rev. Nucl. Part. Sci. 37, 383 (1987); R.K. Ellis, W.J. Stirling and B.R. Webber, QCD and Collider Physics (1996) (Cambridge Uni. Press); B. Andersson, G. Gustafson, G. Ingelman and T. Sjostrand, Phys. Rep. 97, 31 (1983); B. Andersson, The Lund Model (1997) (Cambridge U. Press) ; L.L. Frankfurt and M.I. Strikman, Phys. Rep. 160, 235 (1988); L.N. Lipatov, ibid. 286, 131 (1997); I. Dremin and J.W. Gary, ibid. 349, 301 (2001); S. Catani, F. Krauss, R. Kuhn, and B.R. Webber, JHEP 0111, 63 (2001); C.F. Berger, T. Kucs and G. Sterman, Phys. Rev. D68, 014012 (2003); A. Hebecker, Phys. Rep. 331, 1 (2000); and E.A. De Wolf, J. Phys. G: 28, 1023 (2002).

[11] H.A. Kastrup, Nuc. Phys. B1, 309 (1967); H. Gemmel and H.A. Kastrup, Z. Phys. 229, 321(1969); Nuc. Phys. B14, 566(1969).

[12] Mathematica, Version 4.2, Wolfram Research, Inc.(Champaign, IL). 
[13] Fractional Statistics and Anyon Superconductivity, ed. F. Wilczek (1990) (World Sci. Pub. Co., Singapore); and A. Khare, Fractional Statistics and Quantum Theory (1997) (World Sci. Pub. Co., Singapore).

[14] M.G. Albrow and A. Rostovtsev, hep-ph/0009336; V. Khoze, A.D. Martin, and M. Ryskin, Eur. Phys. J. C14, 525(2000); hep-ph/0207313; A. De Roeck, V. Khoze, A.D. Martin, et.al, Eur. Phys. J. C25, 391(2002); CDF Collab., D. Acosta, et.al., hep-ex/0311032; TOTEM Collab., http://totem.web.cern.ch/Totem.

[15] DAMA Collab., R. Bernabei, et.al., Riv. N. Cim. 26, 1 (2003); CDMS Collab., R. Abusaidi, et.al., Phys. Rev. Lett. 84, 5699(2000); EDELWEISS Collab., A. Benoit, et.al., Phys. Lett. B513, 15(2001); IGEX Collab., H. Morales, et.al., ibid., B489, 268(2000); HDMS Collab., H.V. Klapdor-Kleingrothaus, et.al., Nuc. Phys. Proc. Supp. 110, 364(2002); ZEPLIN Collab., J. Dawson, et.al., 110, 109(2002); SIMPLE Collab., J.I. Collar, et.al., New J. Phys. 2, 14(2000); PICASSO Collab., N. Boukhira, et.al., Nuc. Phys. Proc. Supp. 110, 103(2002).

[16] AGASA Collab., M. Takeda, et.al., Astropar. Phys. 19, 447 (2003); Hi Res Collab., D.R. Berman, et.al, Nuc. Phys. Proc. Supp. 117, 106 (2003); AUGER Collab., http://www.auger.org/; and c.f. V.A. Karmanov and A.E. Kurdyavtsev, hep-ph/0207321.

\section{Figure Captions}

Fig. 1a: The probability $P_{2}(1)$ of "2 charge-paraboson-pairs plus 1 positive paraboson" versus $<n>,\left\langle n^{2}>\right.$. This is the more complex $u \leftrightarrow v$ asymmetric case. The solid line is the $p=1$ curve. Near the peak, the fold is the bottom edge of the ribbon. The open-circles are for the non-folded $u \geq v$ region, and the solid-circles for the folded $u \leq v$ region, see discussion in Sec. 2 . 
Fig. 1b: The front projection, or $X Z$-side, of Fig. 1a, so $P_{2}(1)$ versus $\langle n\rangle$.

Fig. 2: The right-side projection, or $Y Z$-side, for the probability $P_{0}(0)$ of "zero chargeparaboson-pairs plus zero additional single charged parabosons" versus $\left\langle n^{2}\right\rangle$. Since $q$ is even, this is the simpler $u \leftrightarrow v$ symmetric case. Only open-circles from the $u \geq v$ region are shown. The fold is the dashed line $u=v$.

Fig. 3a: The probability $P_{1}(0)$ of " 1 charge-paraboson-pair plus zero additional charged parabosons versus $\langle n\rangle,\left\langle n^{2}\right\rangle$. This is also the simpler $u \leftrightarrow v$ symmetric case so only open-circles from the $u \geq v$ region need to be shown. The solid line is the $p=1$ curve. The fold is the dashed line $u=v$.

Fig. 3b: The right projection, or $Y Z$-side, of Fig. 3a, so $P_{1}(0)$ versus $<n^{2}>$.

Fig. 4a: The probability $P_{1}(1)$ of "1 charge-paraboson-pairs plus 1 positive paraboson" versus $<n>,\left\langle n^{2}>\right.$. This case is $u \leftrightarrow v$ asymmetric.

Fig. 4b: The front projection, or $X Z$-side, of Fig. $4 \mathrm{a}$, so $P_{1}(1)$ versus $\langle n\rangle$.

Fig. 4c: A 3D view "back into the origin" of Fig. 4a.

Fig. 4d: A close-up view of the peak of Fig. 4a. Appendix 2 and its figures discuss various $\{u, v\}$ coordinate charts for this asymmetric case.

Fig. 5a: For $P_{1}(1)$ of Figs. 4, the image of the $u \geq v$ "strip" from $u=2$ to $u=3$ shown in Fig. 5b.

Fig. 5b: The domain $u \geq v$ "strip" from $u=2$ to $u=3$ for Fig.5a.

Fig. 6a: For $P_{1}(1)$, the image of the three vertical lines in Fig. 6b.

Fig. 6b: The three vertical lines $u \simeq 2,3,4$ which are mapped to the 2D surface in Fig. 6a. Fig. 7a: For $P_{1}(1)$, the image of the "ring" of points of Fig. 7b. 
Fig. 7b: The domain of points in the "ring" of radii $r_{<}=3$ and $r_{>}=4$ which are mapped into Fig. 7a.

Fig. 7c: A close-up of Fig. 7a.

Fig. 8a: For $P_{1}(1)$, the image of the points in the unit-negative-slope domain in Fig. 8b. The $\{u, v\}$ values shown are a subset from the unit-negative-slope line of points nearest the origin in Fig. 8b. Note the location of the end points $\{4,0\}$ and $\{0,4\}$.

Fig. 8b: The unit-negative-slope domain whose points are mapped into Fig. 8a: 


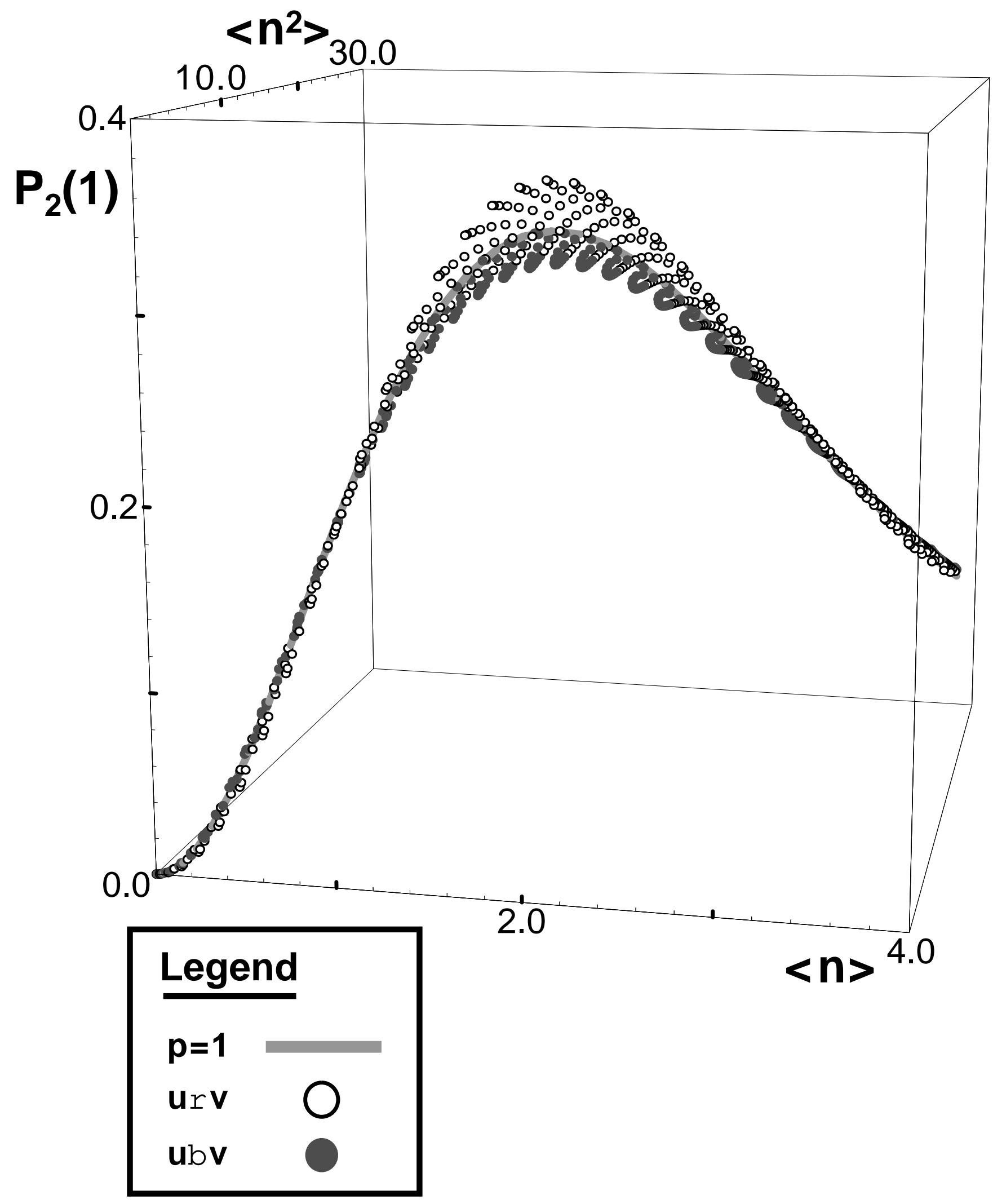




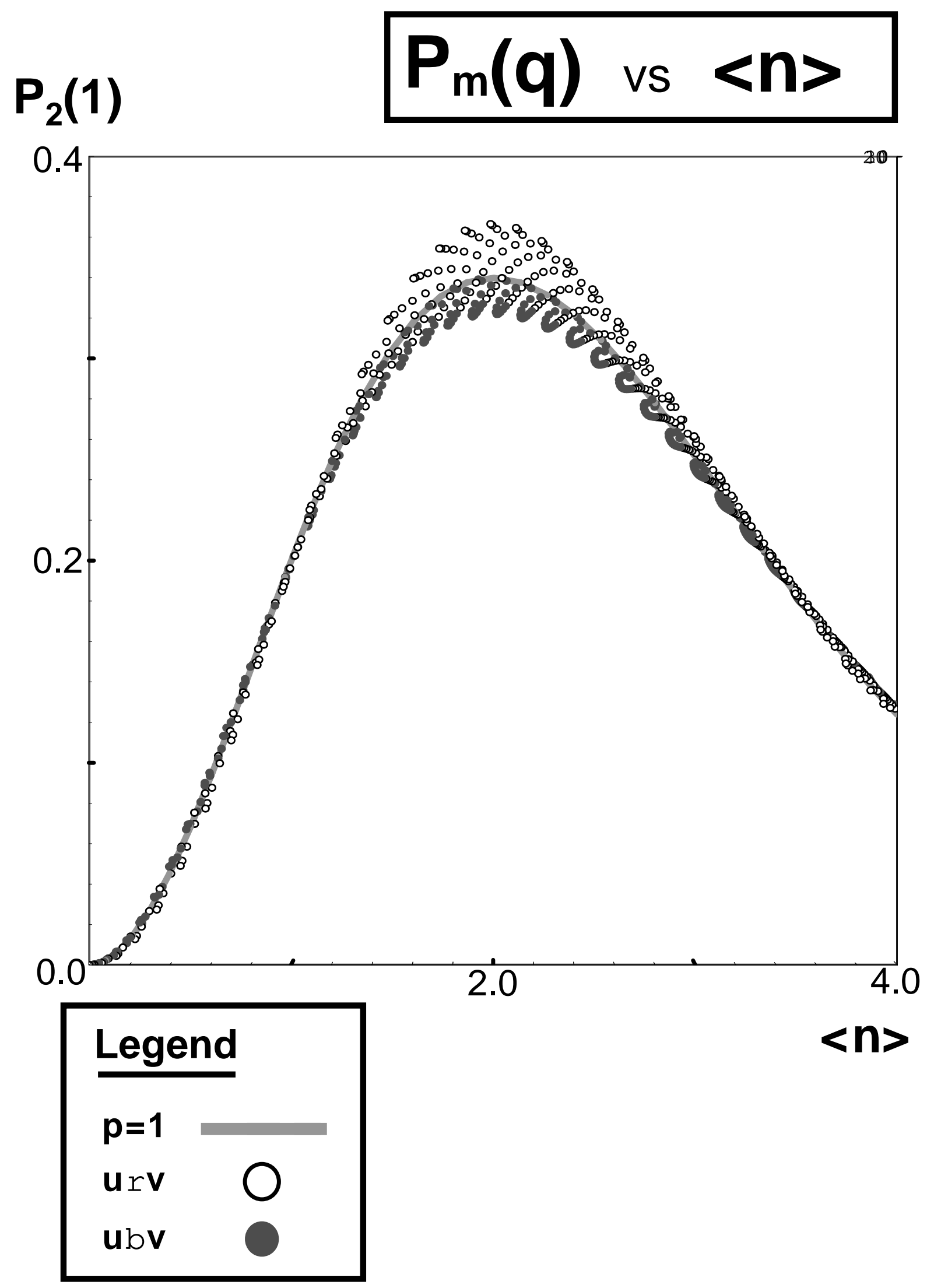




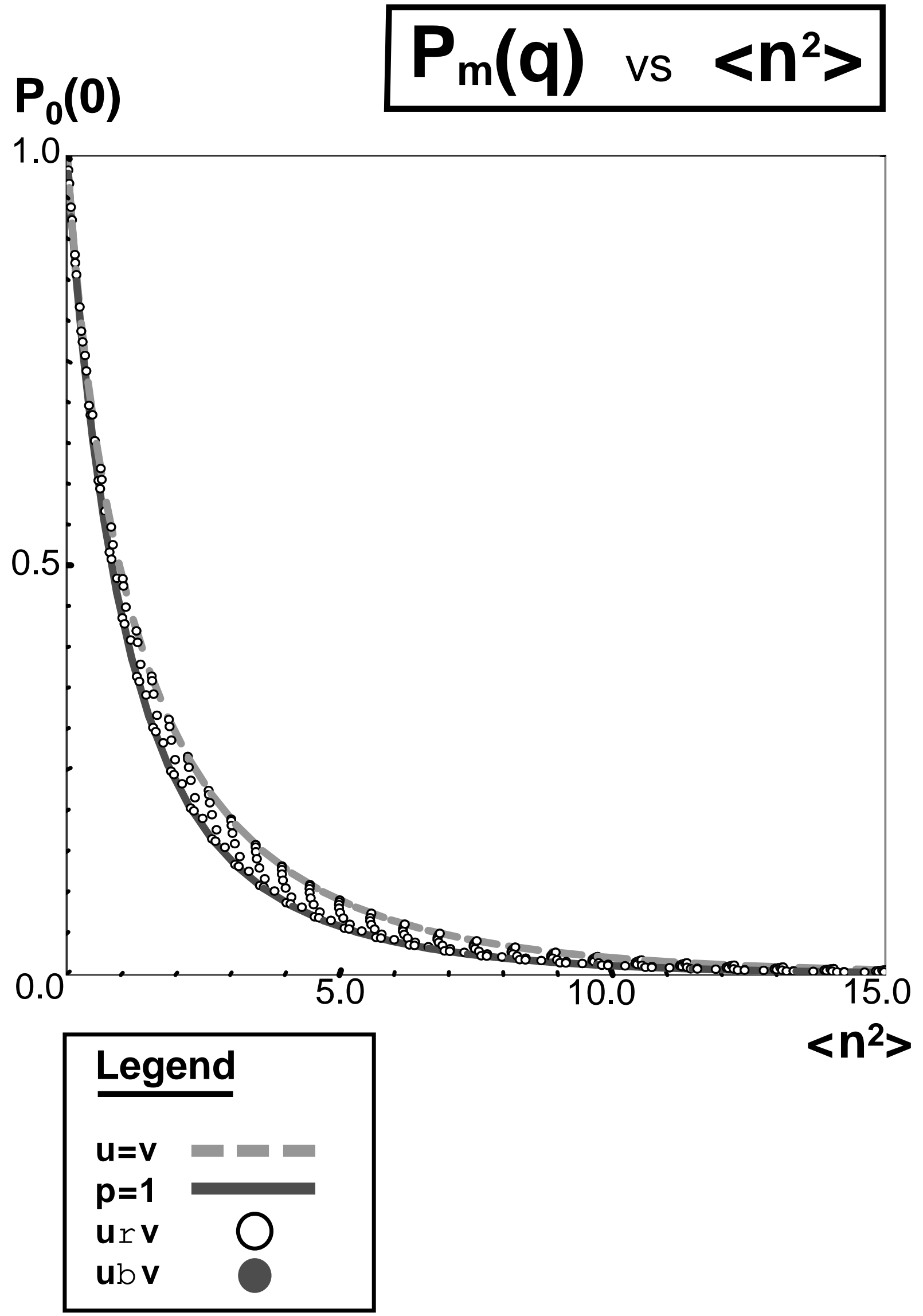




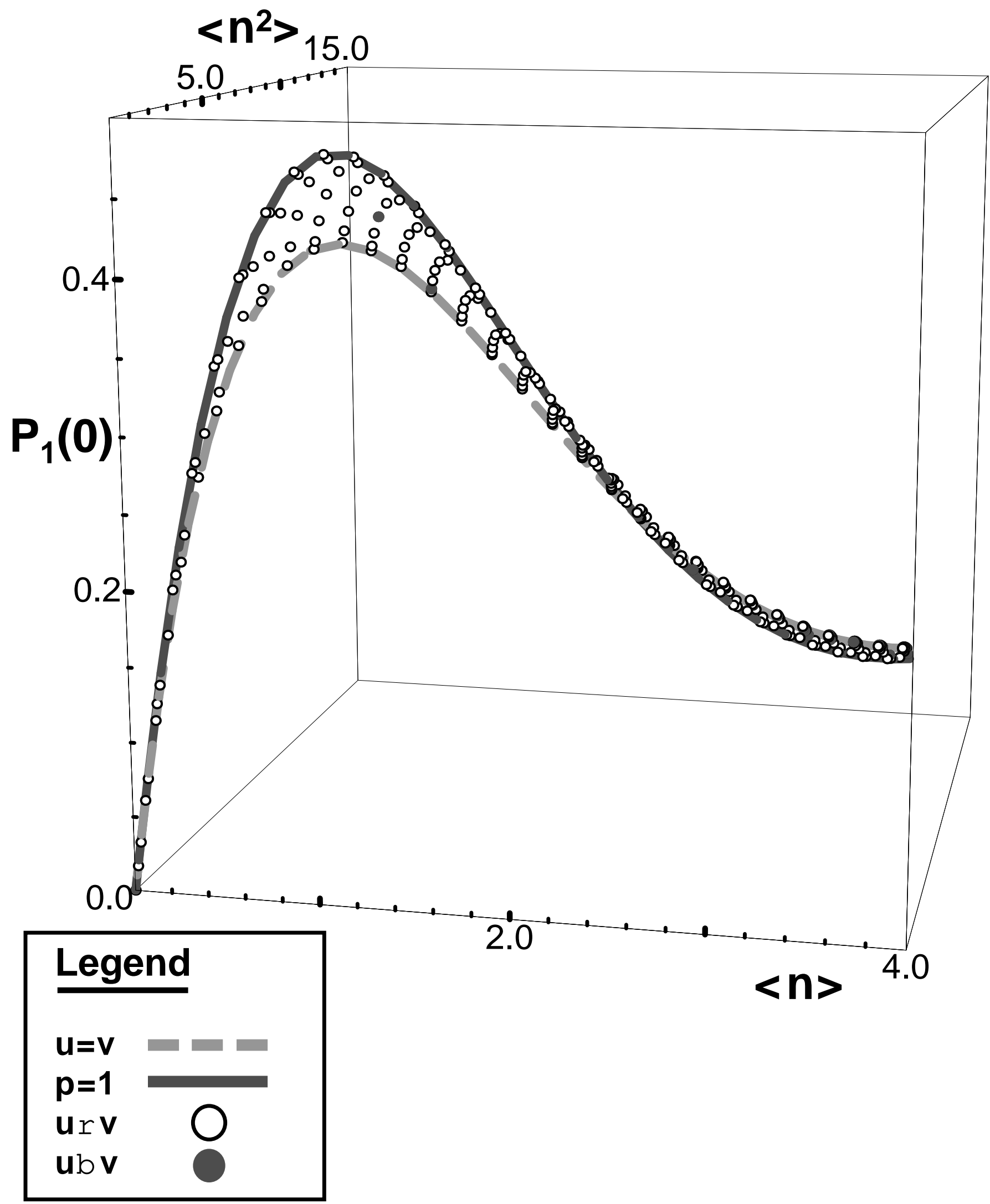


$P_{1}(0)$

\section{$P_{m}(\mathbf{q})$ vs $\left\langle\mathbf{n}^{2}\right\rangle$}

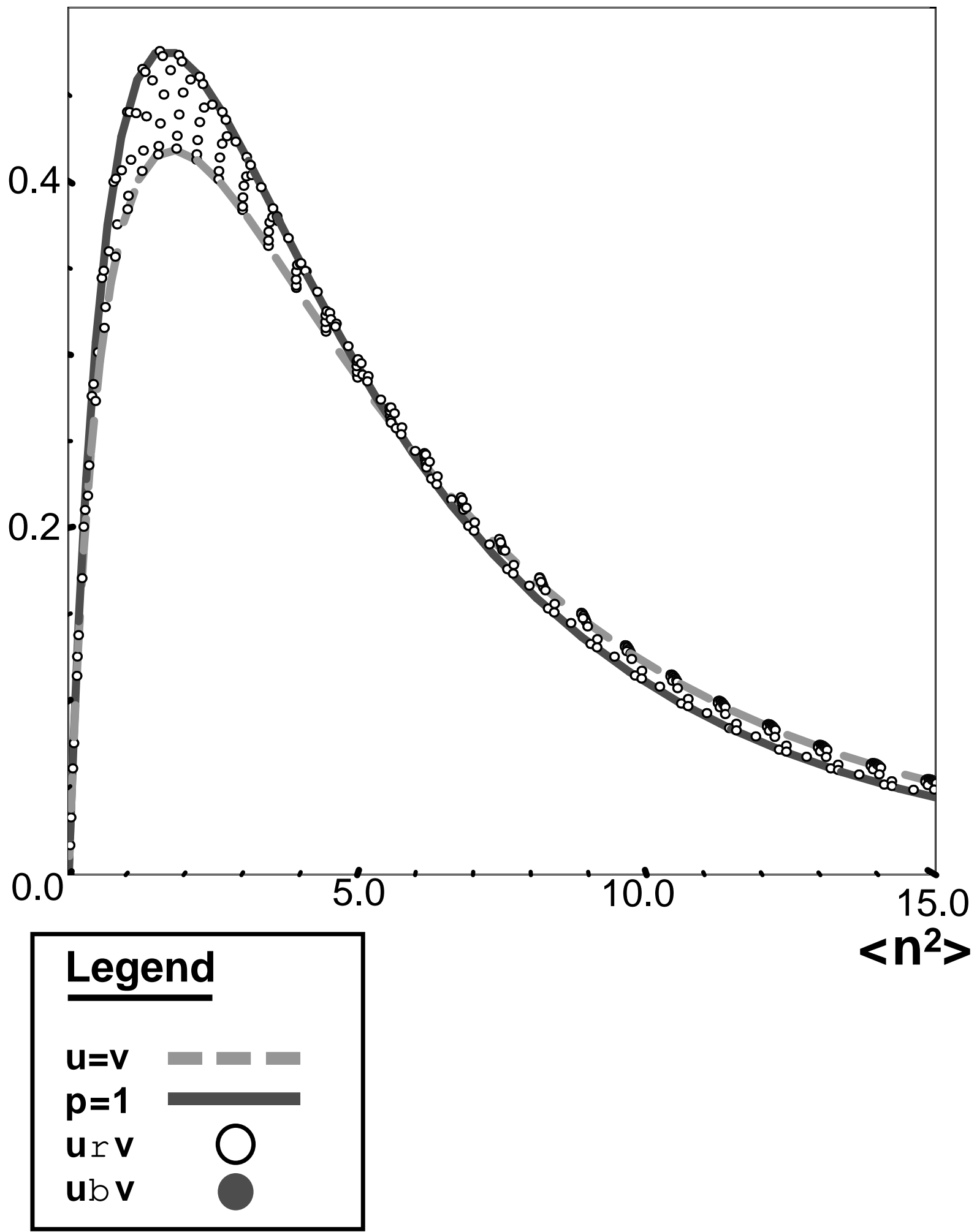




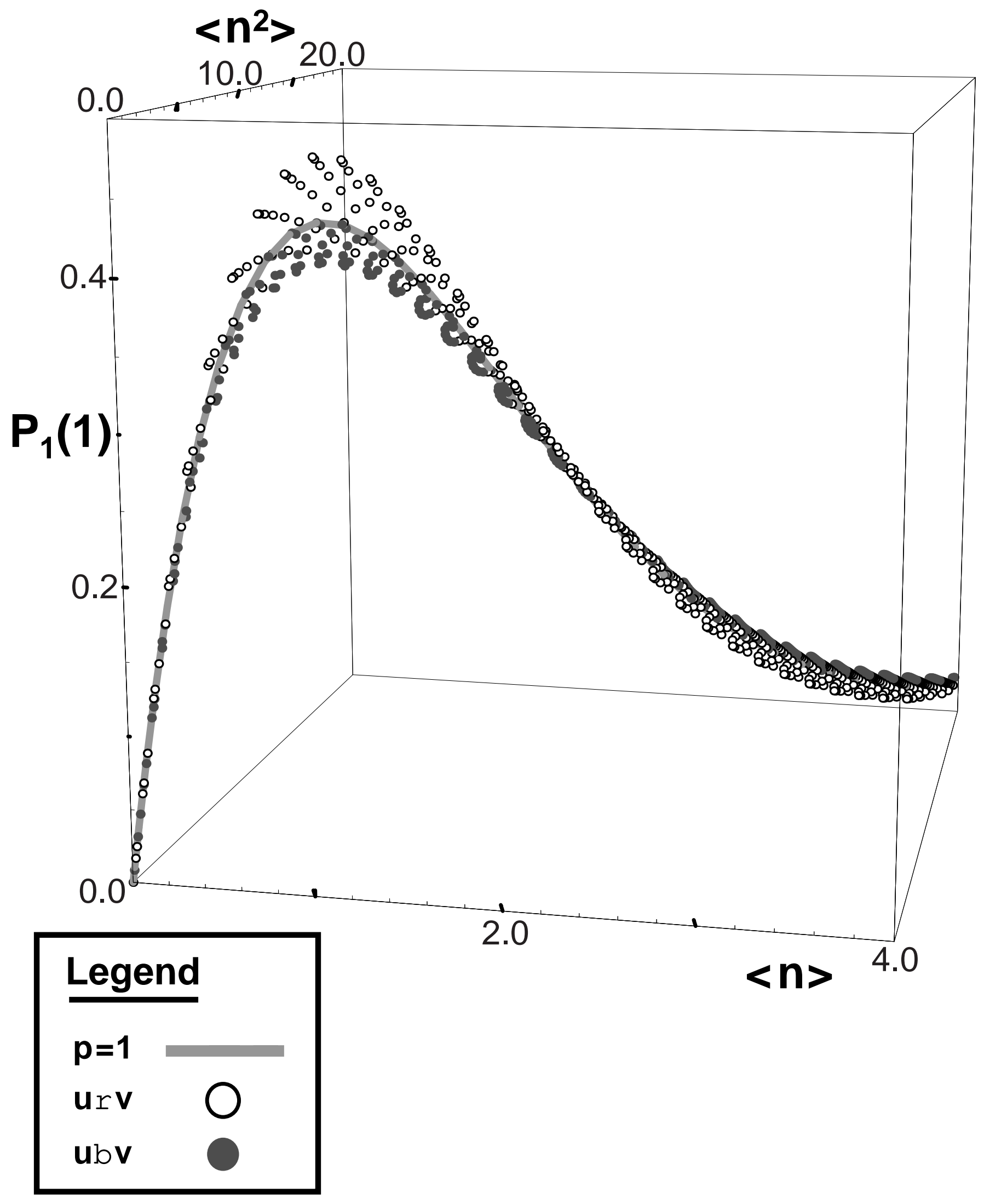


$P_{1}(1)$

\section{$P_{m}(\mathbf{q})$ vs $<\mathrm{n}>$}

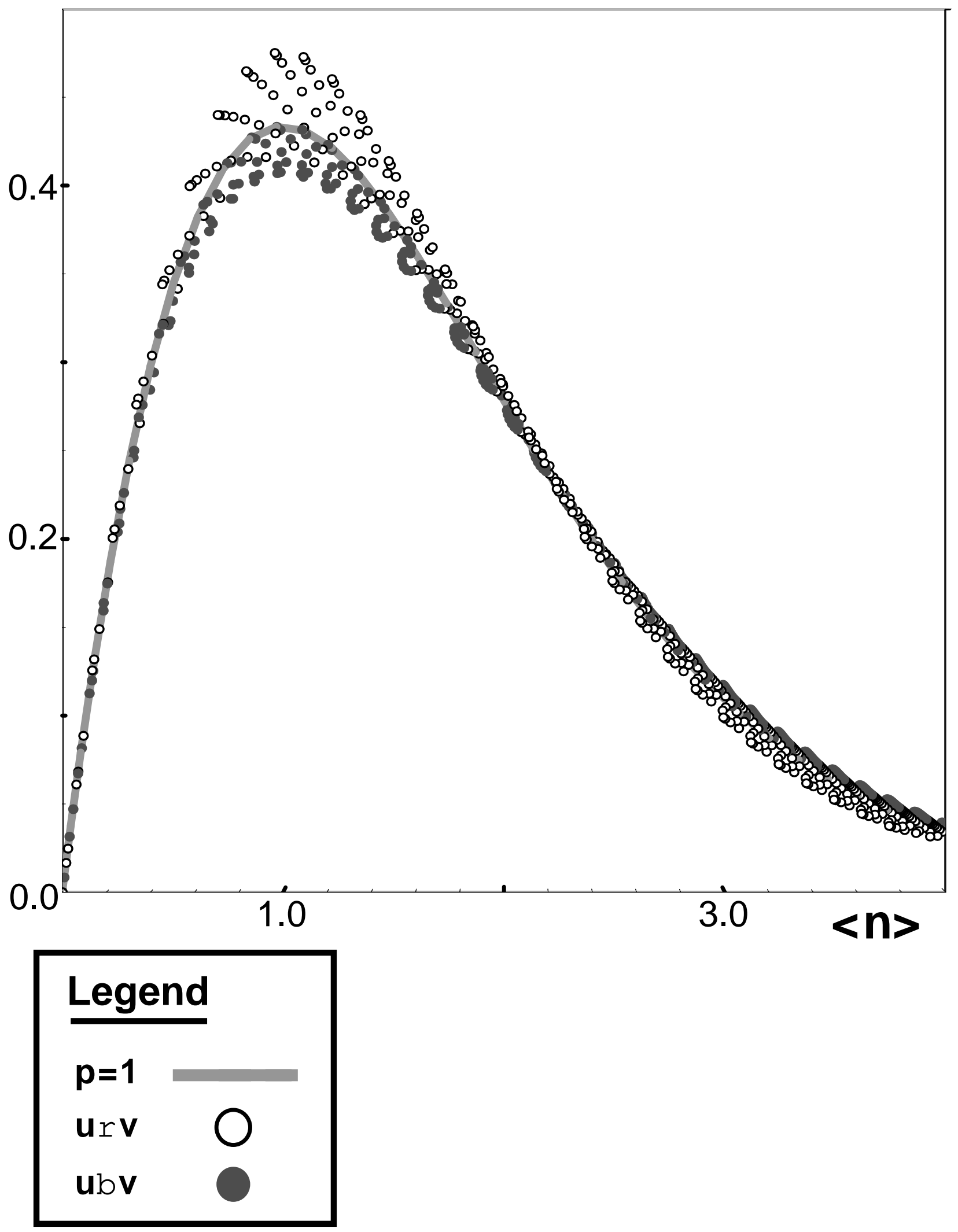




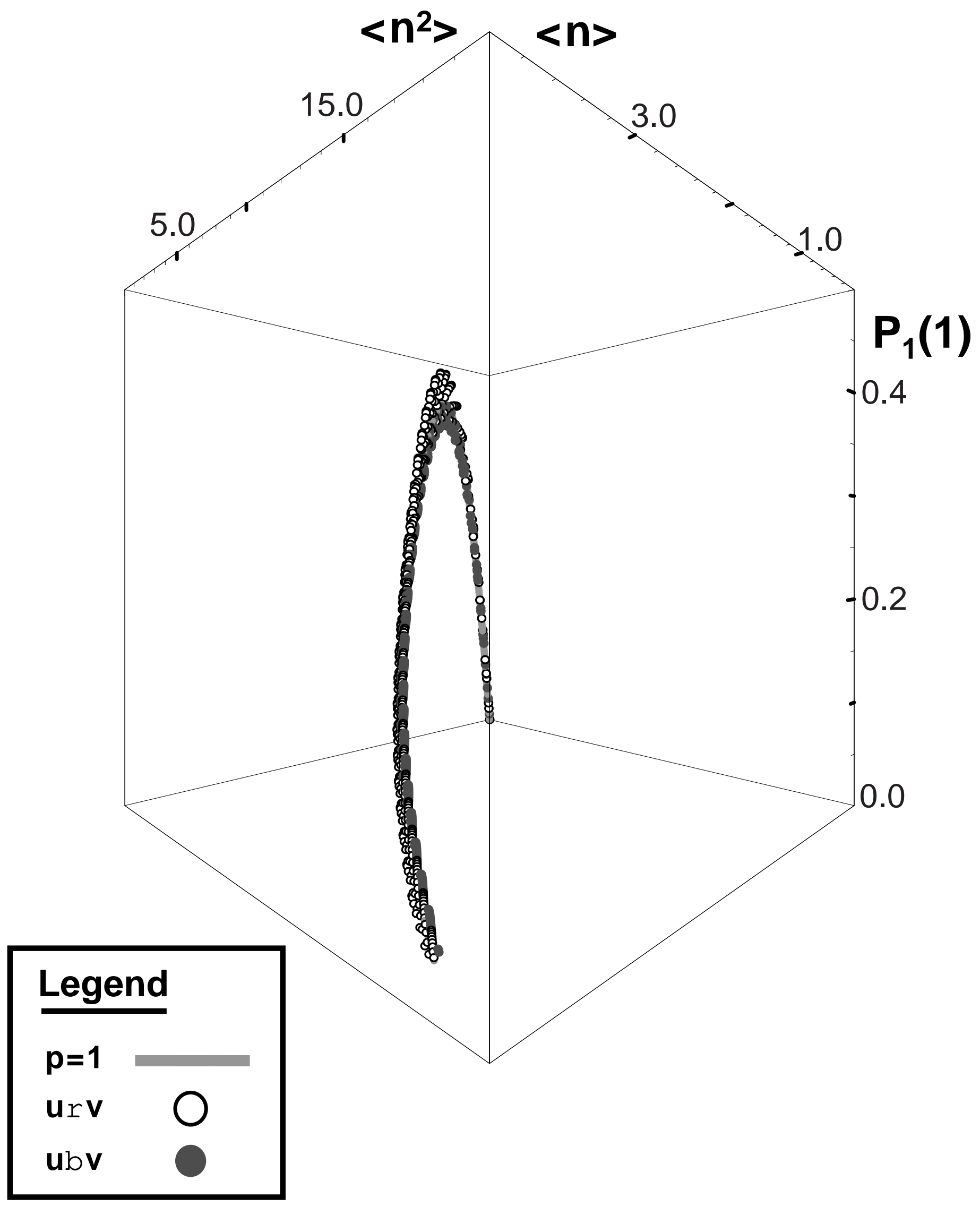




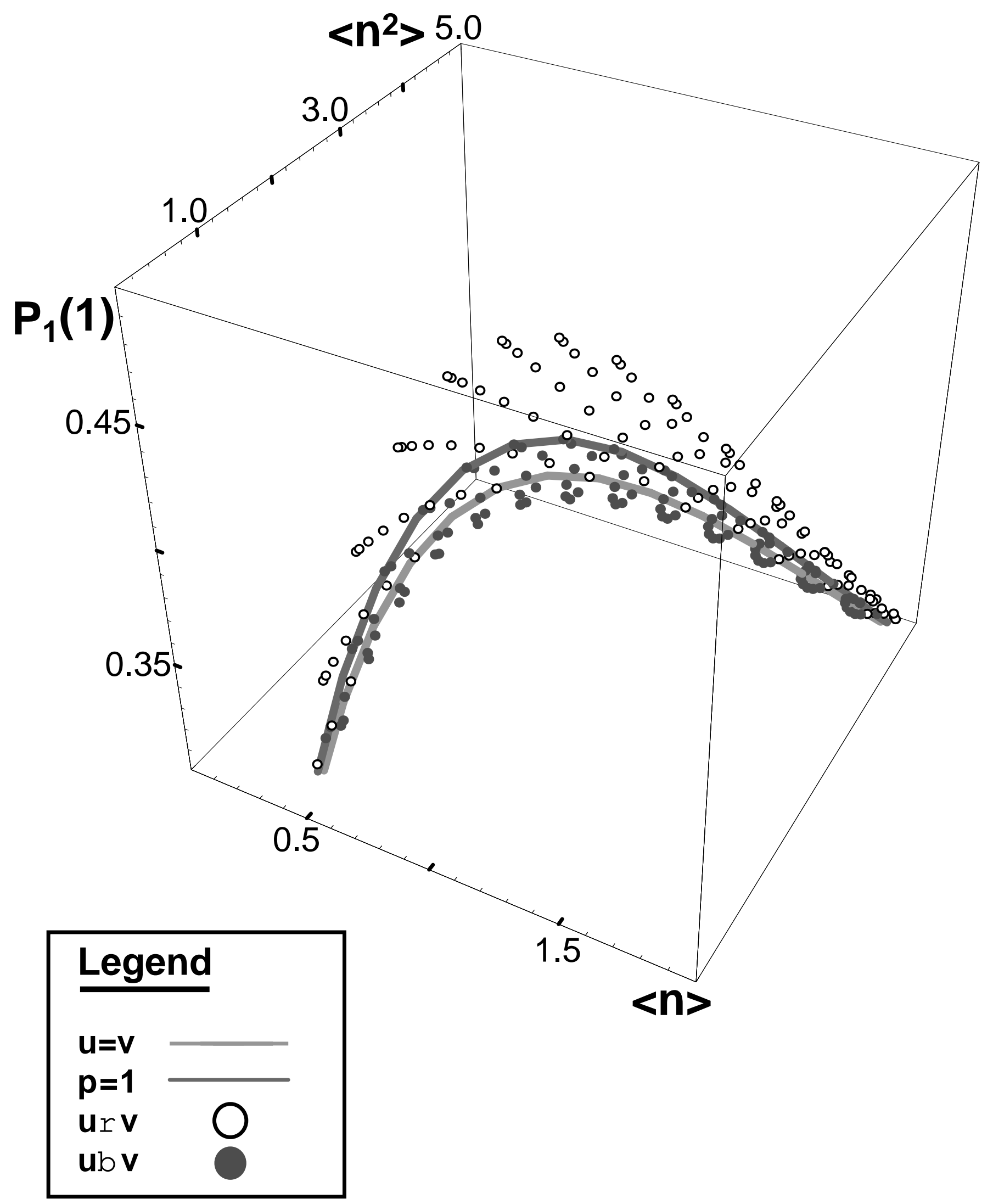




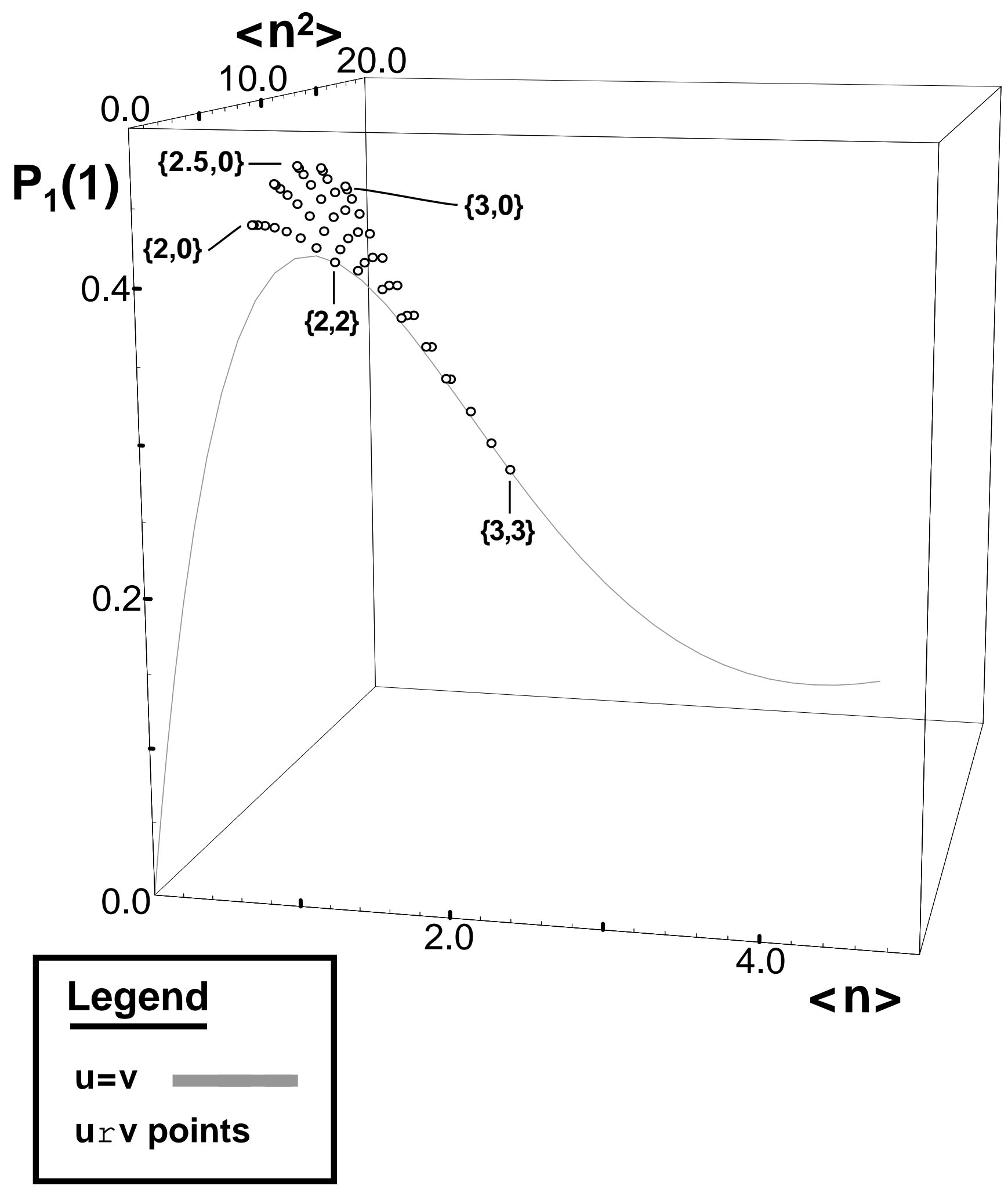




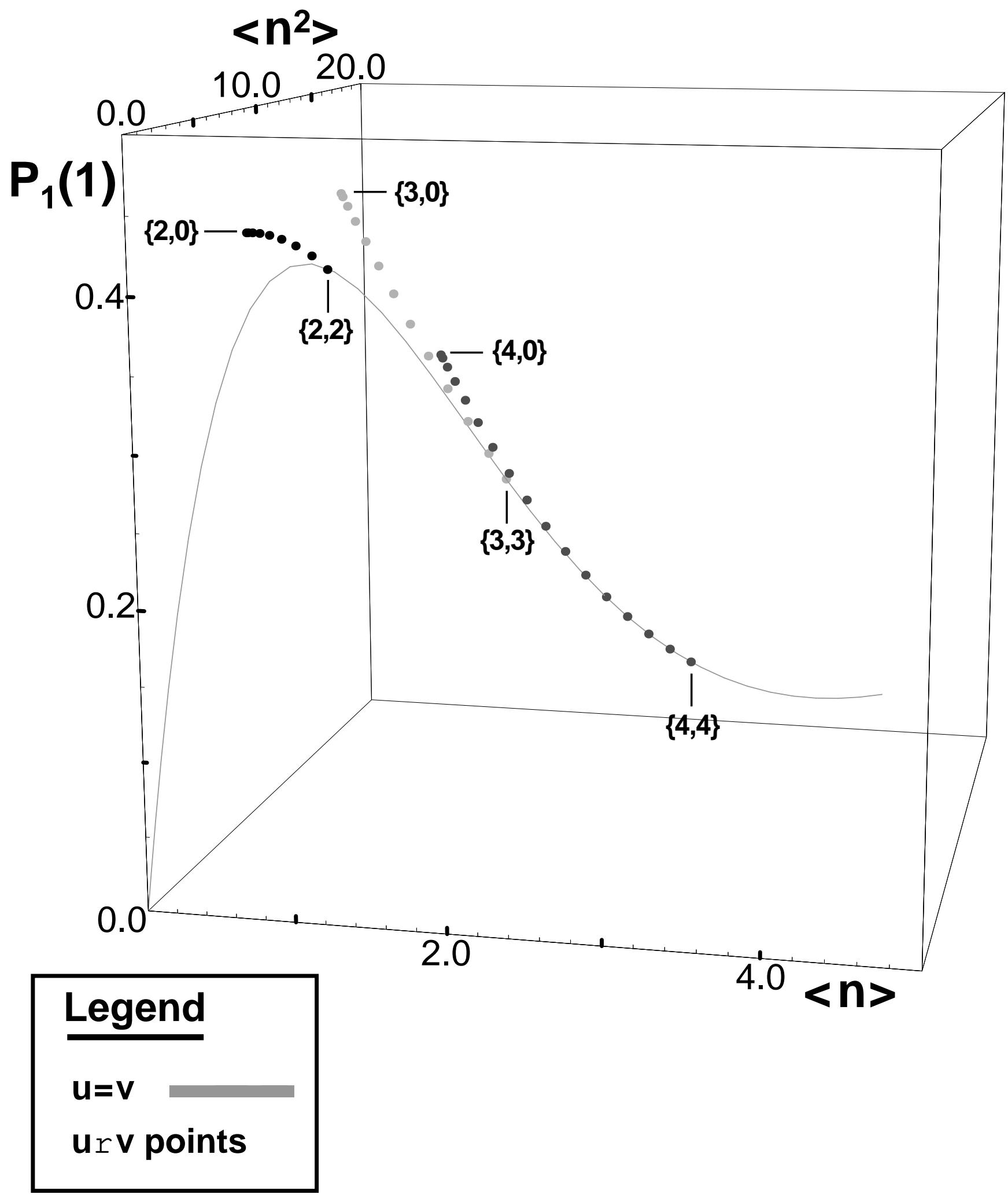




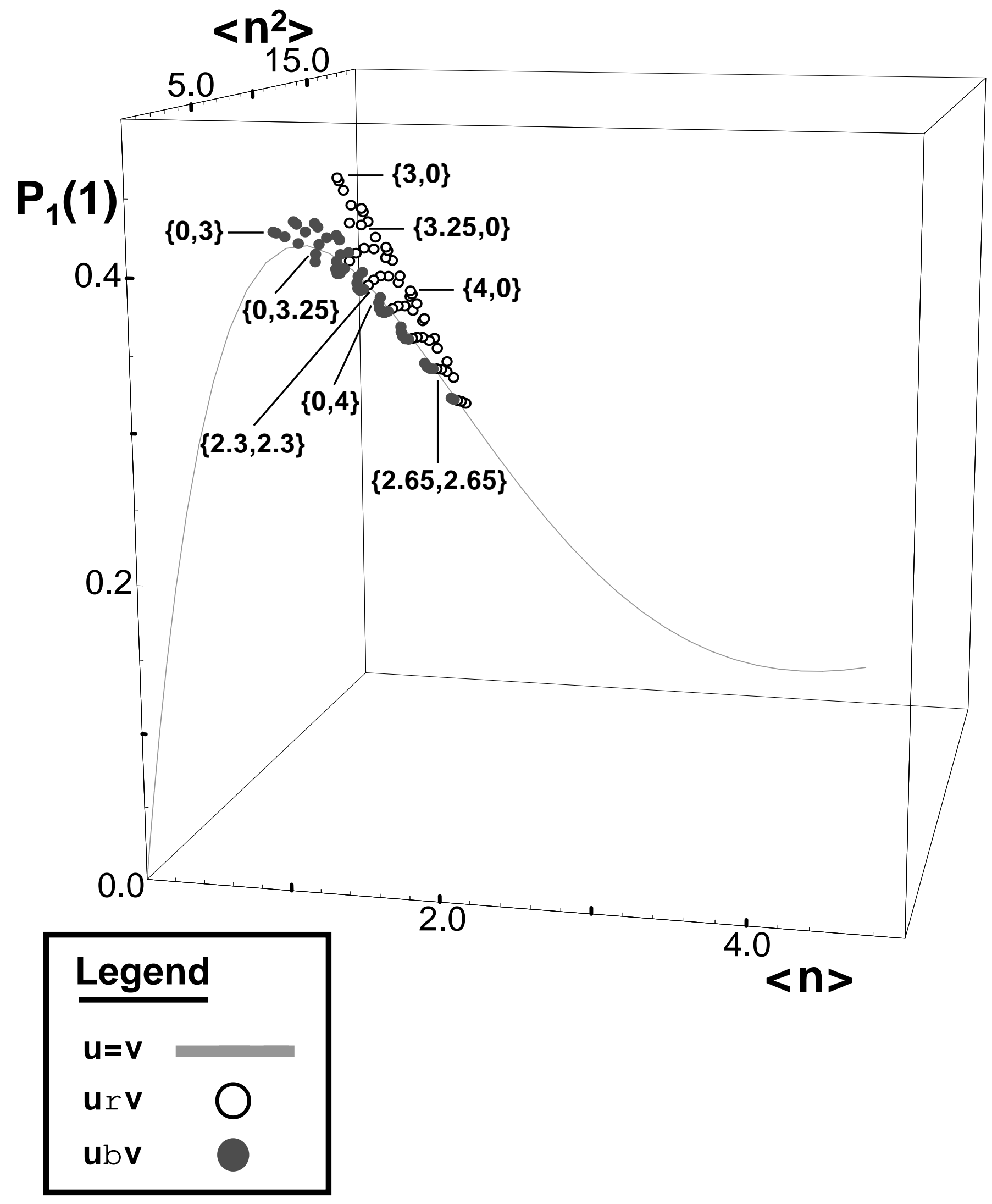




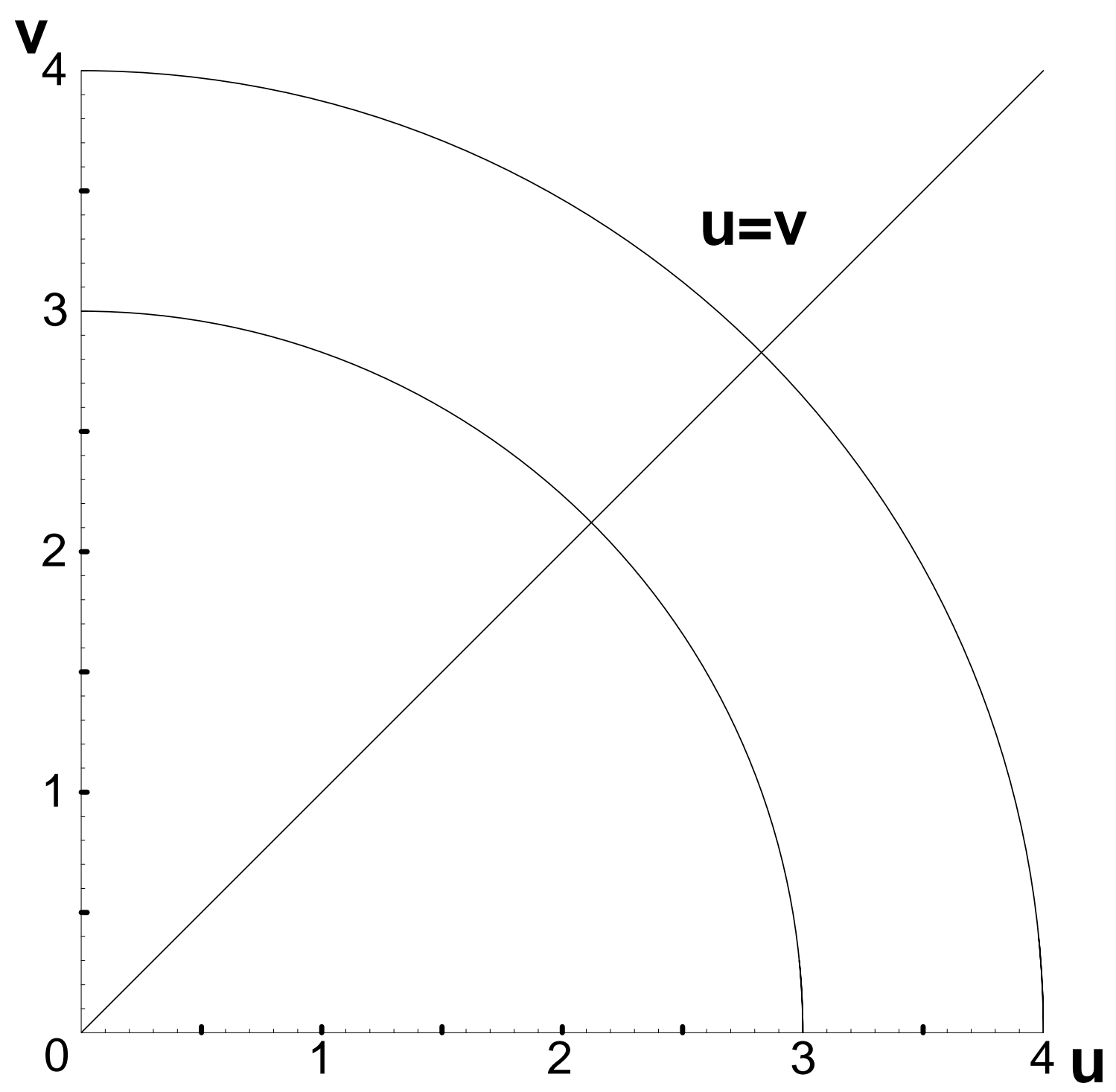




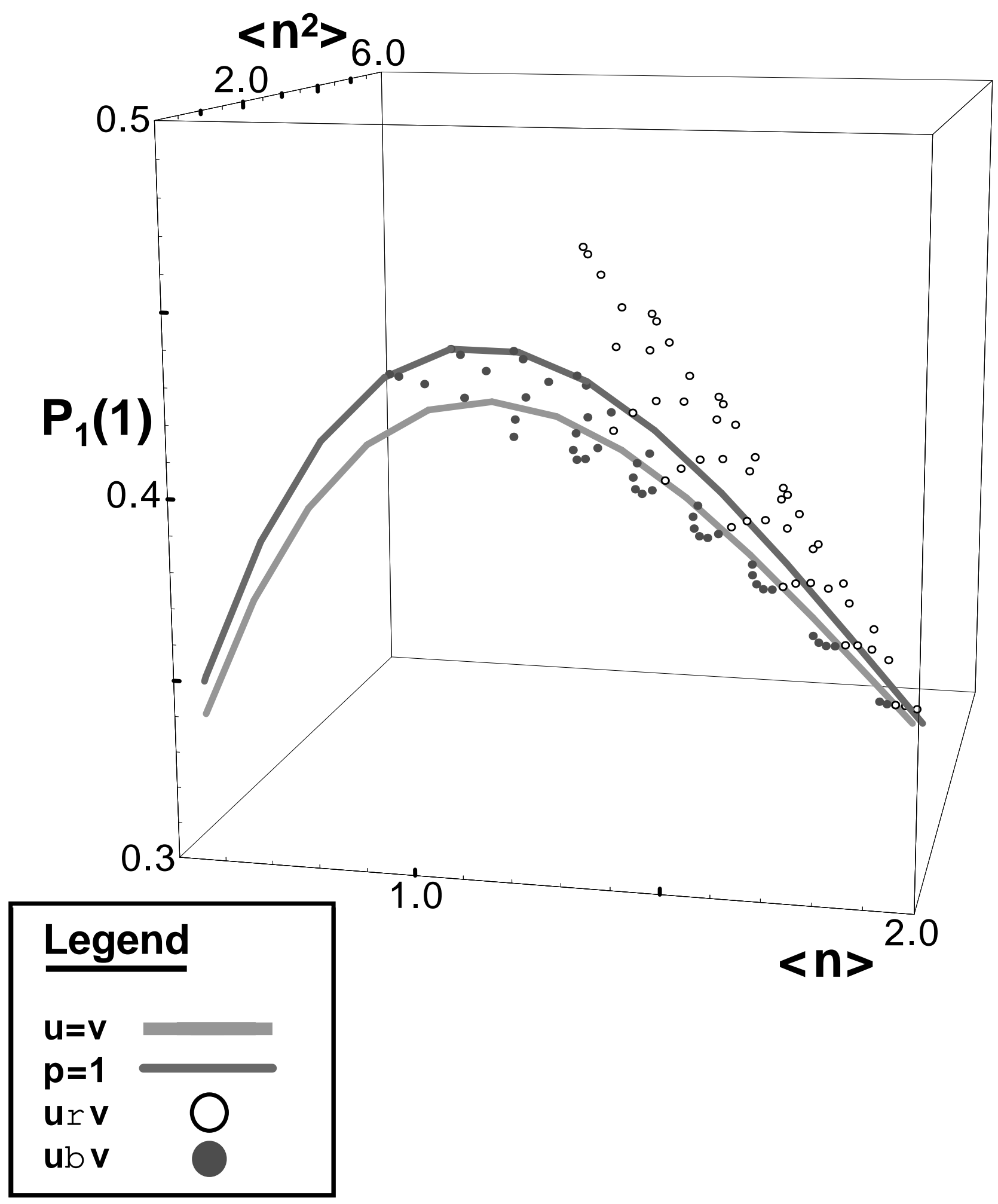




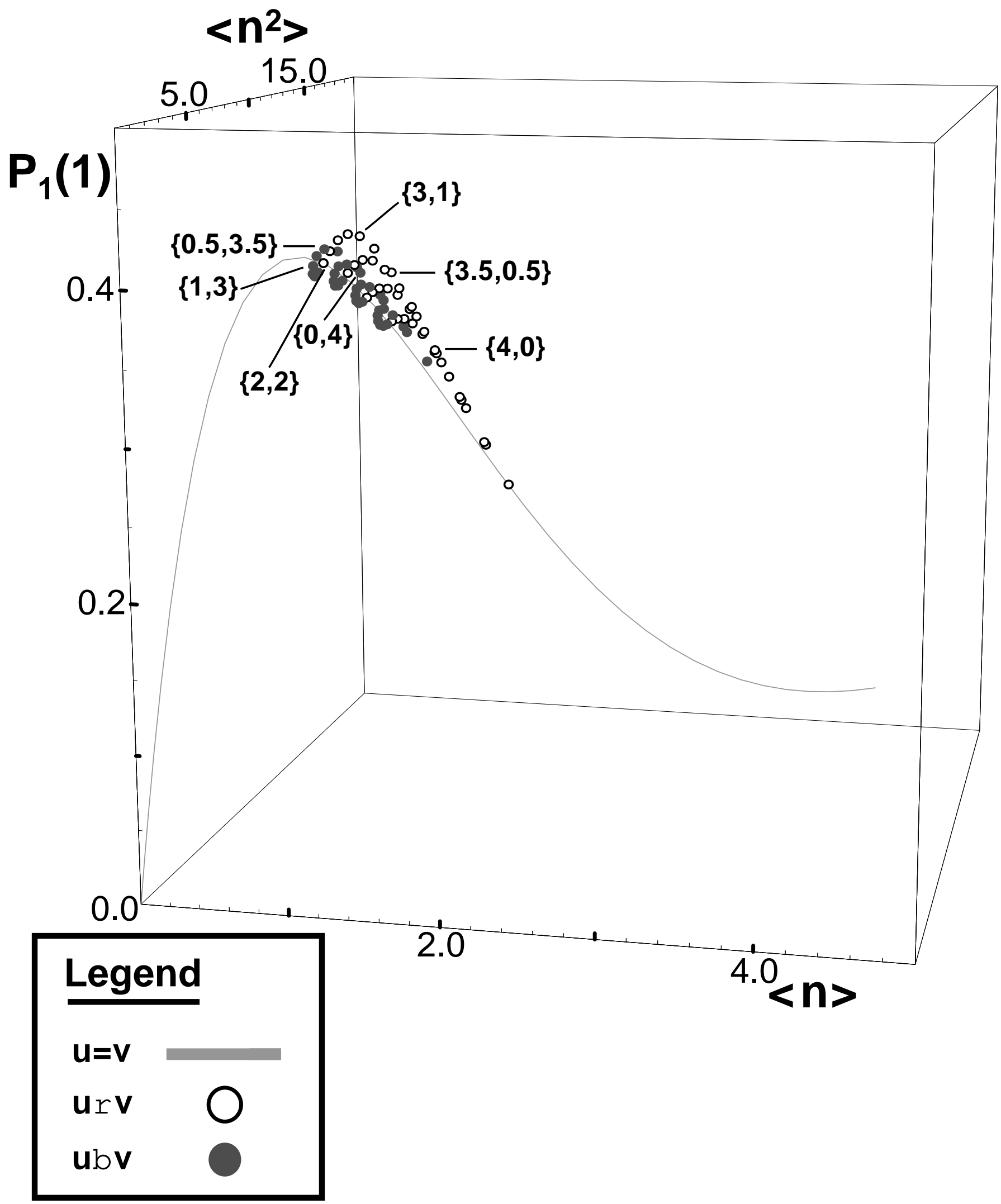




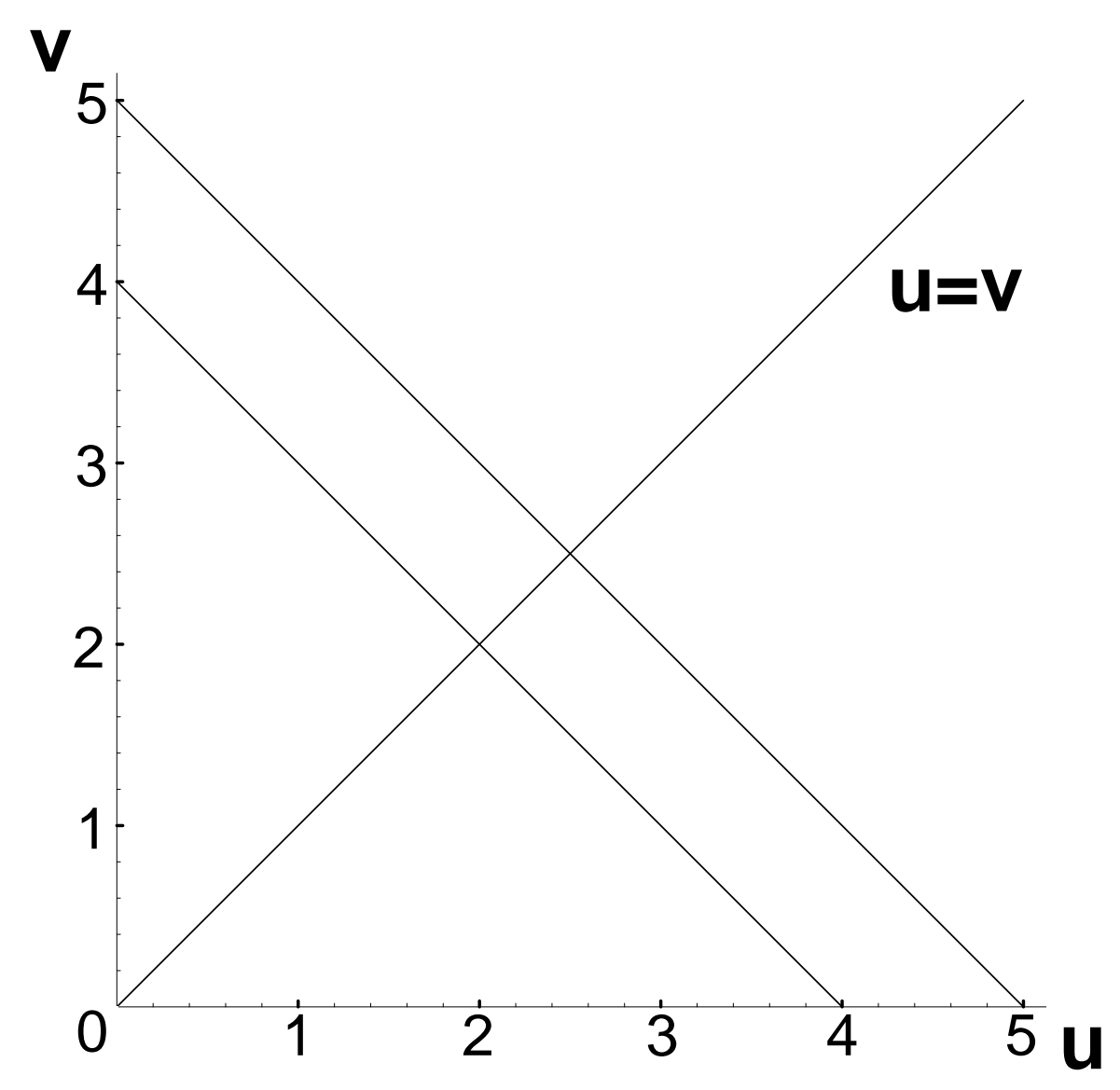

